\title{
New Operations over Interval Valued Intuitionistic Hesitant Fuzzy Set
}

\author{
Said Broumi ${ }^{1, *}$, Florentin Smarandache ${ }^{2}$ \\ ${ }^{1}$ Faculty of Arts and Humanities, Hay El Baraka Ben M'sik Casablanca B.P. 7951, Hassan II University \\ Mohammedia-Casablanca, Morocco \\ ${ }^{2}$ Department of Mathematics, University of New Mexico, 705 Gurley Avenue, Gallup, NM 87301, USA \\ *Corresponding Author: broumisaid78@gmail.com
}

Copyright (C) 2014 Horizon Research Publishing All rights reserved.

\begin{abstract}
Hesitancy is the most common problem in decision making, for which hesitant fuzzy set can be considered as a useful tool allowing several possible degrees of membership of an element to a set. Recently, another suitable means were defined by Zhiming Zhang [1], called interval valued intuitionistic hesitant fuzzy sets, dealing with uncertainty and vagueness, and which is more powerful than the hesitant fuzzy sets. In this paper, four new operations are introduced on interval-valued intuitionistic hesitant fuzzy sets and several important properties are also studied.
\end{abstract}

Keywords Fuzzy Sets, Intuitionistic Fuzzy Set, Hesitant Fuzzy Sets, Interval-Valued Intuitionistic Hesitant Fuzzy Set, Interval Valued Intuitionistic Fuzzy Sets

\section{Introduction}

In recent decades, several types of sets, such as fuzzy sets [2], interval-valued fuzzy sets [3], intuitionistic fuzzy sets [4, 5], interval-valued intuitionistic fuzzy sets [6], type 2 fuzzy sets [7, 8], type n fuzzy sets [7], and hesitant fuzzy sets [9], neutrosophic sets, have been introduced and investigated widely. The concept of intuitionistic fuzzy sets, was introduced by Atanassov [4, 5]; it is interesting and useful in modeling several real life problems.

An intuitionistic fuzzy set (IFS for short) has three associated defining functions, namely the membership function, the non-membership function and the hesitancy function. Later, Atanassov and Gargov provided in [6] what they called interval-valued intuitionistic fuzzy sets theory (IVIFS for short), which is a generalization of both interval valued fuzzy sets and intuitionistic fuzzy sets. Their concept is characterized by a membership function and a non-membership function whose values are intervals rather than real number. IVIFS is more powerful in dealing with vagueness and uncertainty than IFS.

Recently, Torra and Narukawa [9] and Torra [10] proposed the concept of hesitant fuzzy sets, a new generalization of fuzzy sets, which allows the membership of an element of a set to be represented by several possible values. They also discussed relationships among hesitant fuzzy sets and other generalizations of fuzzy sets such as intuitionistic fuzzy sets, type-2 fuzzy sets, and fuzzy multisets. Some set theoretic operations such as union, intersection and complement on hesitant fuzzy sets have also been proposed by Torra [9]. Hesitant fuzzy sets can be used as an efficient mathematical tool for modeling people's hesitancy in daily life than the other classical extensions of fuzzy sets. We'll further study the interval valued intuitionistic hesitant fuzzy sets. Xia and Xu [11] made an intensive study of hesitant fuzzy information aggregation techniques and their applications in decision making. They also defined some new operations on hesitant fuzzy sets based on the interconnection between hesitant fuzzy sets and the interval valued intuitionistic fuzzy sets. To aggregate the hesitant fuzzy information under confidence levels, Xia et al. [12] developed a series of confidence induced hesitant fuzzy aggregations operators. Further, Xia and Xu $[13,14]$ gave a detailed study on distance, similarity and correlation measures for hesitant fuzzy sets and hesitant fuzzy elements respectively. $\mathrm{Xu}$ et al. [15] developed several series of aggregation operators for interval valued intuitionistic hesitant fuzzy information such as: the interval valued intuitionistic fuzzy weighted arithmetic aggregation (IIFWA), the interval valued intuitionistic fuzzy ordered weighted aggregation (IIFOWA) and the interval valued intuitionistic fuzzy hybrid aggregation (IIFHA) operator. Wei and Wang [16], Xu et al. [17] introduced the interval valued intuitionistic fuzzy weighted geometric (IIFWG) operator, the interval valued intuitionistic fuzzy ordered weighted geometric (IIFOWG) operator and the interval valued intuitionistic fuzzy hybrid geometric (IIFHG) operator. Recently, Zhiming Zhang [1] have proposed the concept of interval valued intuitionistic hesitant fuzzy set, study their some basic properties and developed several series of aggregation operators for interval valued intuitionistic hesitant fuzzy environment and have applied them to solve multi-attribute group decision making 
problems.

In this paper, our aim is to propose four new operations on interval valued intuitionistic hesitant fuzzy sets and study their properties.

Therefore, the rest of the paper is set out as follows. In Section 2, some basic definitions related to intuitionistic fuzzy sets, hesitant fuzzy sets and interval valued intuitionistic hesitant fuzzy set are briefly discussed. In Section 3, four new operations on interval valued intuitionistic hesitant fuzzy sets have been proposed and some properties of these operations are proved. In section 4 , we conclude the paper.

\section{Preliminaries}

In this section, we give below some definitions related to intuitionistic fuzzy sets, interval valued intuitionistic fuzzy sets, hesitant fuzzy set and interval valued hesitant fuzzy sets.

\section{Definition 2.1. $[4,5]$ (Set operations on IFS)}

Let IFS(X) denote the family of all intuitionistic fuzzy sets defined on the universe $\mathrm{X}$, and let $\alpha, \beta \in \operatorname{IFS}(\mathrm{X})$ be given as

$$
\alpha=\left(\mu_{\alpha}, v_{\alpha}\right), \beta=\left(\mu_{\beta}, v_{\beta}\right) .
$$

Then nine set operations are defined as follows:

(i) $\quad \alpha^{c}=\left(v_{\alpha}, \mu_{\alpha}\right)$;

(ii) $\quad \alpha \cup \beta=\left(\max \left(\mu_{\alpha}, \mu_{\beta}\right), \min \left(v_{\alpha}, v_{\beta}\right)\right)$;

(iii) $\quad \alpha \cap \beta=\left(\min \left(\mu_{\alpha}, \mu_{\beta}\right), \max \left(v_{\alpha}, v_{\beta}\right)\right)$;

(iv) $\quad \alpha \oplus \beta=\left(\mu_{\alpha}+\mu_{\beta}-\mu_{\alpha} \mu_{\beta}, v_{\alpha} v_{\beta}\right)$;

(v) $\quad \alpha \otimes \beta=\left(\mu_{\alpha} \mu_{\beta}, v_{\alpha}+v_{\beta}-v_{\alpha} v_{\beta}\right)$;

(vi) $\quad \alpha @ \beta=\left(\frac{\mu_{\alpha}+\mu_{\beta}}{2}, \frac{v_{\alpha}+v_{\beta}}{2}\right)$;

(vii) $\quad \alpha \$ \beta=\left(\sqrt{\mu_{\alpha} \mu_{\beta}}, \sqrt{v_{\alpha} v_{\beta}}\right)$;

(viii) $\quad \alpha \# \beta=\left(\frac{2 \mu_{\alpha} \mu_{\beta}}{\mu_{\alpha}+\mu_{\beta}}, \frac{2 v_{\alpha} v_{\beta}}{v_{\alpha}+v_{\beta}}\right)$;

(ix) $\quad \alpha * \beta=\left(\frac{\mu_{\alpha}+\mu_{\beta}}{2\left(\mu_{\alpha} \mu_{\beta}+1\right)}, \frac{v_{\alpha}+v_{\beta}}{2\left(v_{\alpha} v_{\beta}+1\right)}\right)$;

In the following, we introduce some basic concepts related to IVIFS.

\section{Definition 2.2. [6] (Interval valued intuitionistic fuzzy sets)}

An Interval valued intuitionistic fuzzy sets (IVIFS ) $\quad \alpha$ in the finite universe $\mathrm{X}$ is expressed by the form

$\alpha=\left\{<\mathrm{x}, \mu_{\alpha}(\mathrm{x}), v_{\alpha}(\mathrm{x})>\mid \mathrm{x} \in \mathrm{X}\right\}$, where $\mu_{\alpha}(\mathrm{x})=\left[\mu_{\alpha}^{-}(\mathrm{x})\right.$, $\left.\mu_{\alpha}^{+}(\mathrm{x})\right] \in[\mathrm{I}]$ is called membership interval of element to IVIFS $\alpha$,while $\left[v_{\alpha}^{-}(\mathrm{x}), v_{\alpha}^{+}(\mathrm{x})\right] \in[\mathrm{I}]$ is the nonmembership interval of that element to the set $\alpha$, with the condition $0 \leq \mu_{\alpha}^{+}(\mathrm{x})+v_{\alpha}^{+}(\mathrm{x}) \leq 1$ must hold for any $\mathrm{x} \in$ $\mathrm{X}$.

For convenience, the lower and upper bounds of $\mu_{\alpha}(\mathrm{x})$ and $v_{\alpha}(\mathrm{x})$ are denoted by $\mu_{\alpha}^{-}, \mu_{\alpha}^{+}, v_{\alpha}^{-}, v_{\alpha}^{+}$, respectively. Thus, the IVIFS $\alpha$ may be concisely expressed as

$$
\alpha=\left(\mu_{\alpha}, v_{\alpha}\right)=\left\{<\mathrm{X},\left[\mu_{\alpha}^{-}, \mu_{\alpha}^{+}\right],\left[v_{\alpha}^{-}, v_{\alpha}^{+}\right]>\mid \mathrm{x} \in \mathrm{X}\right\}
$$

Where $0 \leq \mu_{\alpha}^{+}+v_{\alpha}^{+} \leq 1$

\section{Definition $2.3[9,11]$}

Let $\mathrm{X}$ be a fixed set. A hesitant fuzzy set (HFS) on $\mathrm{X}$ is in terms of a function that when applied to $\mathrm{X}$ returns a subset of $[0,1]$ the HFS is expressed by a mathematical symbol

$$
\mathrm{E}=\left\{<\mathrm{x}, h_{E}(x)>\mid \mathrm{x} \in \mathrm{X}\right\}
$$

where $h_{E}(x)>$ is a set of some values in $[0,1]$, denoting the possible membership degree of the element $\mathrm{x} \in \mathrm{X}$ to the set $E$. For convenience, Xia and $\mathrm{Xu}$ [11] called $\mathrm{h}=h_{E}(x) \mathrm{a}$ hesitant fuzzy element (HFE) and $H$ be the set of all HFEs.

Given three HFEs represented by $h, h_{1}$, and $h_{2}$, Torra [9] defined some operations on them, which can be described as:

1) $\quad h^{c}=\{1-\gamma \mid \gamma \in h\}$

2) $\quad h_{1} \cup h_{2}=\left\{\max \left(\gamma_{1}, \gamma_{2}\right) \mid \gamma_{1} \in h_{1}, \gamma_{2} \in h_{2}\right\}$

3) $\quad h_{1} \cap h_{2}=\left\{\min \left(\gamma_{1}, \gamma_{2}\right) \mid \gamma_{1} \in h_{1}, \gamma_{2} \in h_{2}\right\}$

Furthermore, in order to aggregate hesitant fuzzy information, Xia and $\mathrm{Xu}$ [11] defined some new operations on the HFEs $h, h_{1}$, and $h_{2}$ :

1) $\quad h_{1} \oplus h_{2}=\left\{\gamma_{1}+\gamma_{2}-\gamma_{1} \gamma_{2} \mid \gamma_{1} \in h_{1}, \gamma_{2} \in h_{2}\right\}$

2) $\quad h_{1} \otimes h_{2}=\left\{\gamma_{1} \gamma_{2} \mid \gamma_{1} \in h_{1}, \gamma_{2} \in h_{2}\right\}$

3) $\quad h^{\lambda}=\left\{\gamma^{\lambda} \mid \gamma \in h\right\}$

4) $\quad \lambda \mathrm{h}=\left\{1-(1-\gamma)^{\lambda} \mid \gamma \in h\right\}$

\section{Definition 2.4 [1] (Interval valued intuitionistic hesitant fuzzy sets)}

Let $\mathrm{X}$ be a fixed set, an interval-valued intuitionistic hesitant fuzzy set (IVIHFS) on $X$ is given in terms of a function that when applied to X returns a subset of $\Omega$. The IVIHFS is expressed by a mathematical symbol

$$
\tilde{E}=\left\{<\mathrm{x}, h_{\tilde{E}}(x)>\mid \mathrm{x} \in \mathrm{X}\right\}
$$

where $h_{\tilde{E}}(x)$ is a set of some IVIFNs in X, denoting the possible membership degree intervals and non-membership degree intervals of the element $\mathrm{x} \in \mathrm{X}$ to the set $\tilde{E}$.

For convenience, an interval-valued intuitionistic hesitant fuzzy element (IVIHFE) is denoted by $\tilde{h}=h_{\tilde{E}}(x)$ and $\tilde{h}$ be the set of all IVIHFEs. If $\alpha \in \tilde{h}$, then an IVIFN can be denoted by $\alpha=\left(\mu_{\alpha}, v_{\alpha}\right)=\left(\left[\mu_{\alpha_{1}}^{-}, \mu_{\alpha_{1}}^{+}\right],\left[v_{\alpha_{1}}^{-}, v_{\alpha_{1}}^{+}\right]\right)$.

For any $\in \tilde{h}$, if $\alpha$ is a real number in $[0,1]$, then $\tilde{h}$ reduces to a hesitant fuzzy element (HFE) [9]; if $\alpha$ is a closed subinterval of the unit interval, then $\tilde{h}$ reduces to an interval-valued hesitant fuzzy element (IVHFE)[1]; if $\alpha$ is an intuitionistic fuzzy number (IFN), then $\tilde{h}$ reduces to an intuitionistic hesitant fuzzy element (IHFE). Therefore, HFEs, IVHFEs, and IHFEs are special cases of IVIHFEs.

\section{Definition 2.5. $[1,9]$}

Given three IVIHFEs represented by $\tilde{h}, \tilde{h}_{1}$, and $\tilde{h}_{2}$, one defines some operations on them, which can be described as: $\tilde{h}^{c}=\left\{\alpha^{c} \mid \alpha \in \tilde{h}\right\}=\left\{\left(\left[v_{\alpha}^{-}, v_{\alpha}^{+}\right],\left[\mu_{\alpha}^{-}, \mu_{\alpha}^{+}\right]\right) \mid \alpha \in \tilde{h}\right\}$, $\tilde{h}_{1} \cup \tilde{h}_{2}=\left\{\max \left(\alpha_{1}, \alpha_{2}\right) \mid \alpha_{1} \in \tilde{h}_{1}, \alpha_{2} \in \tilde{h}_{2}\right\}$ 


$$
\begin{array}{cl}
=\left\{\left(\left[\max \left(\mu_{\alpha_{1}}^{-}, \mu_{\alpha_{2}}^{-}\right), \max \left(\mu_{\alpha_{1}}^{+}, \mu_{\alpha_{2}}^{+}\right)\right],\left[\min \left(v_{\alpha_{1}}^{-}, v_{\alpha_{2}}^{-}\right),\right.\right.\right. & \left.\left.\left.\left.\mu_{\alpha_{1}}^{+} \mu_{\alpha_{2}}^{+}\right],\left[v_{\alpha_{1}}^{-} v_{\alpha_{2}}^{-}, v_{\alpha_{1}}^{+} v_{\alpha_{2}}^{+}\right] \mid \alpha_{1} \in \tilde{h}_{1}, \alpha_{2} \in \tilde{h}_{2}\right)\right)\right\} \\
\left.\left.\left.\min \left(v_{\alpha_{1}}^{+}, v_{\alpha_{2}}^{+}\right)\right]\right) \mid \alpha_{1} \in \tilde{h}_{1}, \alpha_{2} \in \tilde{h}_{2}\right\}, & \tilde{h}_{1} \otimes \tilde{h}_{2}==\left\{\left(\left[\mu_{\alpha_{1}}^{-} \mu_{\alpha_{2}}^{-}, \mu_{\alpha_{1}}^{+} \mu_{\alpha_{2}}^{+}\right],\left[v_{\alpha_{1}}^{-}+v_{\alpha_{2}}^{-}-\right.\right.\right. \\
\tilde{h}_{1} \cap \tilde{h}_{2}=\left\{\min \left(\alpha_{1}, \alpha_{2}\right) \mid \alpha_{1} \in \tilde{h}_{1}, \alpha_{2} \in \tilde{h}_{2}\right\} & \left.\left.v_{\alpha_{1}}^{-} v_{\alpha_{2}}^{-}, v_{\alpha_{1}}^{+}+v_{\alpha_{2}}^{+}-v_{\alpha_{1}}^{+} v_{\alpha_{2}}^{+}\right] \mid \alpha_{1} \in \tilde{h}_{1}, \alpha_{2} \in \tilde{h}_{2}\right\} \\
=\left\{\left(\left[\operatorname { m i n } \left(\mu_{\alpha_{1}}^{-}\right.\right.\right.\right. & \lambda \tilde{h} \\
\left.\left.\left.\mu_{\alpha_{2}}^{-}\right), \min \left(\mu_{\alpha_{1}}^{+}, \mu_{\alpha_{2}}^{+}\right)\right],\left[\max \left(v_{\alpha_{1}}^{-}, v_{\alpha_{2}}^{-}\right), \max \left(v_{\alpha_{1}}^{+}, v_{\alpha_{2}}^{+}\right)\right]\right) \mid \alpha_{1} \in & =\left\{\left(\left[1-\left(1-\mu_{\alpha}^{-}\right)^{\lambda}, 1-\right.\right.\right. \\
\left.\tilde{h}_{1}, \alpha_{2} \in \tilde{h}_{2}\right\}, & \left.\left.\left.\left(1-\mu_{\alpha}^{+}\right)^{\lambda}\right],\left[\left(v_{\alpha}^{-}\right)^{\lambda},\left(v_{\alpha}^{+}\right)^{\lambda}\right]\right) \mid \alpha \in \tilde{h}\right\} \\
\tilde{h}_{1} \oplus \tilde{h}_{2}=\left\{\left(\left(\left[\mu_{\alpha_{1}}^{-}+\mu_{\alpha_{2}}^{-}-\mu_{\alpha_{1}}^{-} \mu_{\alpha_{2}}^{-}, \mu_{\alpha_{1}}^{+}+\mu_{\alpha_{2}}^{+}-\right.\right.\right.\right. &
\end{array}
$$

\section{Four New Operations on IVIHFEs}

\section{Definition 3.1}

Let $\tilde{h}_{1}$ and $\tilde{h}_{2} \in \operatorname{IVIHFE}(\mathrm{X})$, we propose the following operations on IVIHFEs as follows: $\tilde{h}_{1} @ \tilde{h}_{2}=\left\{\left(\left[\frac{\mu_{\alpha_{1}}+\mu_{\alpha_{2}}^{-}}{2}, \frac{\mu_{\alpha_{1}}^{+}+\mu_{\alpha_{2}}^{+}}{2}\right],\left[\frac{v_{\alpha_{1}}+v_{\alpha_{2}}^{-}}{2}, \frac{v_{\alpha_{1}}^{+}+v_{\alpha_{2}}^{+}}{2}\right] \mid \alpha_{1} \in \tilde{h}_{1}, \alpha_{2} \in \tilde{h}_{2}\right\}\right.$

2) $\quad \tilde{h}_{1} \$ \tilde{h}_{2}=\left\{\left(\left[\sqrt{\mu_{\alpha_{1}}^{-} \mu_{\alpha_{2}}^{-}}, \sqrt{\mu_{\alpha_{1}}^{+} \mu_{\alpha_{2}}^{+}}\right],\left[\sqrt{v_{\alpha_{1}}^{-} v_{\alpha_{2}}^{-}}, \sqrt{v_{\alpha_{1}}^{+} v_{\alpha_{2}}^{+}}\right] \mid \alpha_{1} \in \tilde{h}_{1}, \alpha_{2} \in \tilde{h}_{2}\right\}\right.$

3) $\quad \tilde{h}_{1} \# \tilde{h}_{2}=\left\{\left(\left[\frac{2 \mu_{\alpha_{1}} \mu_{\alpha_{2}}^{-}}{\mu_{\alpha_{1}}^{-}+\mu_{\alpha_{2}}^{-}}, \frac{2 \mu_{\alpha_{1}}^{+} \mu_{\alpha_{2}}^{+}}{\mu_{\alpha_{1}}^{+}+\mu_{\alpha_{2}}^{+}}\right],\left[\frac{2 v_{\alpha_{1}}^{-} v_{\alpha_{2}}^{-}}{v_{\alpha_{1}}^{-}+v_{\alpha_{2}}^{-}}, \frac{\left.2 v_{\alpha_{1}}^{+} v_{\alpha_{2}}^{+}\right]_{1}}{v_{\alpha_{1}}^{+}+v_{\alpha_{2}}^{+}}\right] \mid \alpha_{1} \in \tilde{h}_{1}, \alpha_{2} \in \tilde{h}_{2}\right\}\right.$

4) $\quad \tilde{h}_{1} * \tilde{h}_{2}=\left\{\left(\left[\frac{\mu_{\alpha_{1}}+\mu_{\alpha_{2}}^{-}}{2\left(\mu_{\alpha_{1}}^{-} \mu_{\alpha_{2}}^{-}+1\right)}, \frac{\mu_{\alpha_{1}}^{+}+\mu_{\alpha_{2}}^{+}}{2\left(\mu_{\alpha_{1}}^{+} \mu_{\alpha_{2}}^{+}+1\right)}\right],\left[\frac{v_{\alpha_{1}}+v_{\alpha_{2}}^{-}}{2\left(v_{\alpha_{1}}^{-} v_{\alpha_{2}}^{-}+1\right)}, \frac{v_{\alpha_{1}}^{+}+v_{\alpha_{2}}^{+}}{2\left(v_{\alpha_{1}}^{+} v_{\alpha_{2}}^{+}+1\right)}\right] \mid \alpha_{1} \in \tilde{h}_{1}, \alpha_{2} \in \tilde{h}_{2}\right\}\right.$

Obviously, for every two IVIHFEs $\tilde{h}_{1}$ and $\tilde{h}_{2},\left(\tilde{h}_{1} @ \tilde{h}_{2}\right),\left(\tilde{h}_{1} \$ \tilde{h}_{2}\right),\left(\tilde{h}_{1} \# \tilde{h}_{2}\right)$ and $\left(\tilde{h}_{1} * \tilde{h}_{2}\right)$ are also IVIHFEs.

\section{Example 3.2}

Let $\tilde{h}_{1}(\mathrm{x})=\{([0.2,0.3],[0.5,0.6]),([0.5,0.8],[0.1,0.2])\}$ and $\tilde{h}_{2}(\mathrm{x})=\{([0.4,0.6],[0.3,0.4]),([0.3,0.5],[0.1,0.2])$ be two interval valued intuitionistic hesitant fuzzy elements. Then we have

$\left(\tilde{h}_{1} @ \tilde{h}_{2}\right)=\{([0.3,0.45],[0.4,0.5]),([0.4,0.65],[0.1,0.2])\}$

$\left(\tilde{h}_{1} \$ \tilde{h}_{2}\right)=\{([0.28,0.42],[0.38,0.48]),([0.38,0.63],[0.1,0.2])\}$

$\left(\tilde{h}_{1} \# \tilde{h}_{2}\right)=\{([0.26,0.4],[0.37,0.48]),([0.37,0.61],[0.1,0.2])\}$

$\left(\tilde{h}_{1} * \tilde{h}_{2}\right)=\{([0.27,0.38],[0.34,0.40]),([0.34,0.46],[0.09,0.19])\}$

With these operations, several results follow.

\section{Theorem 3.3}

For every $\tilde{h} \in \operatorname{IVIHFE}(\mathrm{X})$, the following are true,

(i) $\quad \tilde{h} @ \tilde{h}=\tilde{h}$;

(ii) $\quad \tilde{h} \$ \tilde{h}=\tilde{h}$;

(iii) $\quad \tilde{h} \# \tilde{h}=\tilde{h}$;

Proof. we prove only (i) (ii) .

(i) Let $\tilde{h} \in$ IVIHFEs

$\tilde{h} @ \tilde{h}=\left\{\left[\frac{\mu_{\alpha}^{-}+\mu_{\alpha}^{-}}{2}, \frac{\mu_{\alpha}^{+}+\mu_{\alpha}^{+}}{2}\right],\left[\frac{v_{\alpha}^{-}+v_{\alpha}^{-}}{2}, \frac{v_{\alpha}^{+}+v_{\alpha}^{+}}{2}\right] \mid \alpha \in \tilde{h}\right\}$

Then, $\tilde{h} @ \tilde{h}=\tilde{h}$ $=\left[\mu_{\alpha}^{-}, \mu_{\alpha}^{+}\right],\left[v_{\alpha}^{-}, v_{\alpha}^{+}\right]$

(ii) Let $\tilde{h} \in$ IVIHFEs

$\tilde{h} \$ \tilde{h}=\left\{\left(\left[\sqrt{\mu_{\alpha}^{-} \mu_{\alpha}^{-}}, \sqrt{\mu_{\alpha}^{+} \mu_{\alpha}^{+}}\right],\left[\sqrt{v_{\alpha}^{-} v_{\alpha}^{-}}, \sqrt{v_{\alpha}^{+} v_{\alpha}^{+}}\right] \mid \alpha \in \tilde{h}\right\}\right.$

Then, $\tilde{h} \$ \tilde{h}=\tilde{h}$ $=\left[\mu_{\alpha}^{-}, \mu_{\alpha}^{+}\right],\left[v_{\alpha}^{-}, v_{\alpha}^{+}\right]$

\section{Theorem 3.4}

For $\tilde{h}_{1}, \tilde{h}_{2} \in$ IVIHFEs,

(i) $\quad \tilde{h}_{1} @ \widetilde{h}_{2}=\widetilde{h}_{2} @ \tilde{h}_{1}$; 
(ii) $\quad \tilde{h}_{1} \$ \widetilde{h}_{2}=\widetilde{h}_{2} \$ \tilde{h}_{1}$;

(iii) $\quad \widetilde{h}_{1} \# \widetilde{h}_{2}=\widetilde{h}_{2} \# \widetilde{h}_{1}$;

(iv) $\tilde{h}_{1} * \widetilde{h}_{2}=\widetilde{h}_{2} * \widetilde{h}_{1}$;

Proof. These also follow from definitions.

\section{Theorem 3.5}

For $\tilde{h}_{1}, \tilde{h}_{2} \in \operatorname{IVIHFE}(\mathrm{X})$,

$\left(\tilde{h}_{1}^{c} @ \tilde{h}_{2}^{c}\right)^{c}=\tilde{h}_{1} @ \tilde{h}_{2}$

Proof. In the following, we prove (i), (ii) and (iii), results (iv), (v) and (vi) can be proved analogously.

$$
\begin{aligned}
\left(\tilde{h}_{1}{ }^{c} @ \tilde{h}_{2}{ }^{c}\right)^{c} & =\left\{\left[v_{\alpha_{1}}^{-}, v_{\alpha_{1}}^{+}\right],\left[\mu_{\alpha_{1}}^{-}, \mu_{\alpha_{1}}^{+}\right] \mid \alpha_{1} \in \tilde{h}_{1}\right\} @\left\{\left[v_{\alpha_{2}}^{-}, v_{\alpha_{2}}^{+}\right],\left[\mu_{\alpha_{2}}^{-}, \mu_{\alpha_{2}}^{+}\right] \mid \alpha_{2} \in \tilde{h}_{2}\right\} \\
\left(\tilde{h}_{1}{ }^{c} @ \tilde{h}_{2}{ }^{c}\right)^{c} & =\left(\left\{\left[v_{\alpha_{1}}^{-}, v_{\alpha_{1}}^{+}\right],\left[\mu_{\alpha_{1}}^{-}, \mu_{\alpha_{1}}^{+}\right] \mid \alpha_{1} \in \tilde{h}_{1}\right\} @\left\{\left[v_{\alpha_{2}}^{-}, v_{\alpha_{2}}^{+}\right],\left[\mu_{\alpha_{2}}^{-}, \mu_{\alpha_{2}}^{+}\right] \mid \alpha_{2} \in \tilde{h}_{2}\right\}\right)^{c} \\
& =\left(\left\{\left[\frac{v_{\alpha_{1}}+v_{\alpha_{2}}^{-}}{2}, \frac{v_{\alpha_{1}}^{+}+v_{\alpha_{2}}^{+}}{2}\right],\left[\frac{\mu_{\alpha_{1}}+\mu_{\alpha_{2}}^{-}}{2}, \frac{\mu_{\alpha_{1}}^{+}+\mu_{\alpha_{2}}^{+}}{2}\right] \mid \alpha_{1} \in \tilde{h}_{1}, \alpha_{2} \in \tilde{h}_{2}\right\}\right)^{c} \\
& =\left(\left\{\left[\frac{\mu_{\alpha_{1}+\mu_{\alpha_{2}}}^{-}}{2}, \frac{\mu_{\alpha_{1}}^{+}+\mu_{\alpha_{2}}^{+}}{2}\right],\left[\frac{v_{\alpha_{1}}+v_{\alpha_{2}}^{-}}{2}, \frac{v_{\alpha_{1}}^{+}+v_{\alpha_{2}}^{+}}{2}\right] \mid \alpha_{1} \in \tilde{h}_{1}, \alpha_{2} \in \tilde{h}_{2}\right\}\right)
\end{aligned}
$$

$=\tilde{h}_{1} @ \widetilde{h}_{2}$

This proves the theorem.

Note 1: One can easily verify that

$$
\begin{array}{ll}
\text { 1. } & \left(\tilde{h}_{1}{ }^{c} \$ \tilde{h}_{2}{ }^{c}\right)^{c} \neq \tilde{h}_{1} \$ \widetilde{h}_{2} \\
\text { 2. } & \left(\tilde{h}_{1}{ }^{c} \# \tilde{h}_{2}{ }^{c}\right)^{c} \neq \tilde{h}_{1} \# \widetilde{h}_{2} \\
\text { 3. } & \left(\tilde{h}_{1}{ }^{c} * \tilde{h}_{2}{ }^{c}\right)^{c} \neq \tilde{h}_{1} * \widetilde{h}_{2}
\end{array}
$$

\section{Theorem 3.6}

For $\tilde{h}_{1}, \tilde{h}_{2}$ and $\tilde{h}_{3} \in \operatorname{IVIHFE}(\mathrm{X})$, we have the following identities:
(i) $\quad\left(\tilde{h}_{1} \cup \tilde{h}_{2}\right) @ \tilde{h}_{3}=\left(\tilde{h}_{1} @ \tilde{h}_{3}\right) \cup\left(\tilde{h}_{2} @ \tilde{h}_{3}\right)$;
(ii) $\quad\left(\tilde{h}_{1} \cap \tilde{h}_{2}\right) @ \tilde{h}_{3}=\left(\tilde{h}_{1} @ \tilde{h}_{3}\right) \cap\left(\tilde{h}_{2} @ \tilde{h}_{3}\right)$
(iii) $\quad\left(\tilde{h}_{1} \cup \tilde{h}_{2}\right) \$ \tilde{h}_{3}=\left(\tilde{h}_{1} \$ \tilde{h}_{3}\right) \cup\left(\tilde{h}_{2} \$ \tilde{h}_{3}\right)$;
(iv) $\quad\left(\tilde{h}_{1} \cap \tilde{h}_{2}\right) \$ \tilde{h}_{3}=\left(\tilde{h}_{1} \$ \widetilde{h}_{3}\right) \cap\left(\tilde{h}_{2} \$ \widetilde{h}_{3}\right)$;
(v) $\quad\left(\left(\tilde{h}_{1} \cup \tilde{h}_{2}\right)\right) \# \tilde{h}_{3}=\left(\tilde{h}_{1} \# \widetilde{h}_{3}\right) \cup\left(\tilde{h}_{2} \# \widetilde{h}_{3}\right)$;
(vi) $\quad\left(\tilde{h}_{1} \cap \tilde{h}_{2}\right) \# \tilde{h}_{3}=\left(\tilde{h}_{1} \# \tilde{h}_{3}\right) \cap\left(\tilde{h}_{2} \# \tilde{h}_{3}\right)$;
(vii) $\quad\left(\tilde{h}_{1} \cup \tilde{h}_{2}\right) * \tilde{h}_{3}=\left(\tilde{h}_{1} * \tilde{h}_{3}\right) \cup\left(\tilde{h}_{2} * \tilde{h}_{3}\right)$;
(viii) $\quad\left(\tilde{h}_{1} \cap \tilde{h}_{2}\right) * \tilde{h}_{3}=\left(\tilde{h}_{1} * \tilde{h}_{3}\right) \cap\left(\tilde{h}_{2} * \tilde{h}_{3}\right)$;
(ix) $\quad\left(\tilde{h}_{1} @ \widetilde{h}_{2}\right) \oplus \tilde{h}_{3}=\left(\tilde{h}_{1} \oplus \widetilde{h}_{3}\right) @\left(\tilde{h}_{2} \oplus \widetilde{h}_{3}\right)$;
(x) $\quad\left(\tilde{h}_{1} @ \tilde{h}_{2}\right) \otimes \tilde{h}_{3}=\left(\tilde{h}_{1} \otimes \tilde{h}_{3}\right) @\left(\tilde{h}_{2} \otimes \tilde{h}_{3}\right)$

Proof. We prove (i), (iii), (v), (vii) and (ix), results (ii), (iv), (vi), (viii) and (x) can be proved analogously Using definitions in 2.3 and 3.1, we have

$\left(\tilde{h}_{1} \cup \tilde{h}_{2}\right) @ \tilde{h}_{3}=\left\{\left[\max \left(\mu_{\alpha_{1}}^{-}, \mu_{\alpha_{2}}^{-}\right), \max \left(\mu_{\alpha_{1}}^{+}, \mu_{\alpha_{2}}^{+}\right)\right],\left[\min \left[\left(\hat{\nu}_{\alpha_{1}}^{-}, v_{\alpha_{2}}^{-}\right), \min \left(v_{\alpha_{1}}^{+}, v_{\alpha_{2}}^{+}\right)\right] \mid \alpha_{1} \in \tilde{h}_{1}, \alpha_{2} \in \tilde{h}_{2}\right\} @\left\{\left[\mu_{\alpha_{3}}^{-}\right.\right.\right.$,

$$
\left.\left.\mu_{\alpha_{3}}^{+}\right],\left[v_{\alpha_{3}}^{-}, v_{\alpha_{3}}^{+}\right] \mid \alpha_{3} \in \tilde{h}_{3}\right\}
$$

$$
=\left\{\left[\frac{\max \left(\mu_{\alpha_{1}}^{-}, \mu_{\alpha_{2}}^{-}\right)+\mu_{\alpha_{3}}^{-}}{2}, \frac{\max \left(\mu_{\alpha_{1}}^{+}, \mu_{\alpha_{2}}^{+}\right)+\mu_{\alpha_{3}}^{+}}{2}\right],\left[\frac{\min \left(v_{\alpha_{1}}^{-}, v_{\alpha_{2}}^{-}\right)+v_{\alpha_{3}}^{-}}{2}, \frac{\min \left(v_{\alpha_{1}}^{+}, v_{\alpha_{2}}^{+}\right)+v_{\alpha_{3}}^{+}}{2}\right] \mid \alpha_{1} \in \tilde{h}_{1}, \alpha_{2} \in \tilde{h}_{2}, \alpha_{3} \in \tilde{h}_{3}\right\}
$$

$=\left\{\left[\max \left(\frac{\mu_{\alpha_{1}}+\mu_{\alpha_{3}}^{-}}{2}, \frac{\mu_{\alpha_{2}}+\mu_{\alpha_{3}}^{-}}{2}\right), \max \left(\frac{\mu_{\alpha_{1}}^{+}+\mu_{\alpha_{3}}^{+}}{2}, \frac{\mu_{\alpha_{2}}^{+}+\mu_{\alpha_{3}}^{+}}{2}\right)\right],\left[\min \left(\frac{v_{\alpha_{1}}^{-}+v_{\alpha_{3}}^{-}}{2}, \frac{v_{\alpha_{2}+v_{\alpha_{3}}^{-}}^{-}}{2}\right), \min \left(\frac{v_{\alpha_{1}}^{-}+v_{\alpha_{3}}^{-}}{2}, \frac{v_{\alpha_{1}}^{+}+v_{\alpha_{3}}^{+}}{2}\right)\right] \mid \alpha_{1} \in\right.$ $=\left(\tilde{h}_{1} @ \tilde{h}_{3}\right) \cup\left(\tilde{h}_{2} @ \tilde{h}_{3}\right)$

This proves (i)

(iii) From definitions in 2.3 and 3.1, we have

$\left(\tilde{h}_{1} \cup \tilde{h}_{2}\right) \$ \tilde{h}_{3}=\left\{\left[\max \left(\mu_{\alpha_{1}}^{-}, \mu_{\alpha_{2}}^{-}\right), \max \left(\mu_{\alpha_{1}}^{+}, \mu_{\alpha_{2}}^{+}\right)\right],\left[\min \left[\left(v_{\alpha_{1}}^{-}, v_{\alpha_{2}}^{-}\right), \min \left(v_{\alpha_{1}}^{+}, v_{\alpha_{2}}^{+}\right)\right] \mid \alpha_{1} \in \tilde{h}_{1}, \alpha_{2} \in \tilde{h}_{2}\right\} \$\left\{\left[\mu_{\alpha_{3}}^{-}\right.\right.\right.$, $\left.\left.\mu_{\alpha_{3}}^{+}\right],\left[v_{\alpha_{3}}^{-}, v_{\alpha_{3}}^{+}\right] \mid \alpha_{3} \in \tilde{h}_{3}\right\}$ 


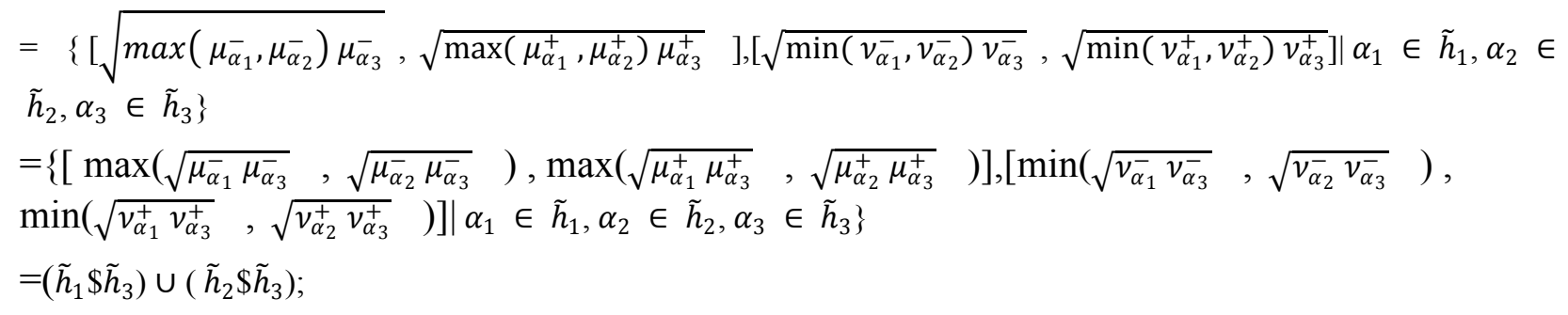

This proves (iii).

(v) Using definitions 2.3 and 3.1, we have

$\left(\left(\tilde{h}_{1} \cup \tilde{h}_{2}\right)\right) \# \tilde{h}_{3}=\left\{\left[\max \left(\mu_{\alpha_{1}}^{-}, \mu_{\alpha_{2}}^{-}\right), \max \left(\mu_{\alpha_{1}}^{+}, \mu_{\alpha_{2}}^{+}\right)\right],\left[\min \left(\left(v_{\alpha_{1}}^{-}, v_{\alpha_{2}}^{-}\right), \min \left(v_{\alpha_{1}}^{+}, v_{\alpha_{2}}^{+}\right)\right] \mid \alpha_{1} \in \tilde{h}_{1}, \alpha_{2} \in \tilde{h}_{2}\right\} \#\left\{\left[\mu_{\alpha_{3}}^{-}\right.\right.\right.$, $\left.\left.\mu_{\alpha_{3}}^{+}\right],\left[v_{\alpha_{3}}^{-}, v_{\alpha_{3}}^{+}\right] \mid \alpha_{3} \in \tilde{h}_{3}\right\}$

$$
\begin{gathered}
=\left\{\left[\frac{2 \max \left(\mu_{\alpha_{1}}, \mu_{\alpha_{2}}\right) \mu_{\alpha_{3}}^{-}}{\max \left(\mu_{\alpha_{1}}^{-}, \mu_{\alpha_{2}}\right)+\mu_{\alpha_{3}}^{-}}, \frac{2 \max \left(\mu_{\alpha_{1}}^{+}, \mu_{\alpha_{2}}^{+}\right) \mu_{\alpha_{3}}^{+}}{\max \left(\mu_{\alpha_{1}}^{+}, \mu_{\alpha_{2}}^{+}\right)+\mu_{\alpha_{2}}^{+}}\right],\left[\frac{2 \min \left[\left[v_{\alpha_{1}}, v_{\alpha_{2}}\right) v_{\alpha_{3}}^{-}\right.}{\min \left[\left(v_{\alpha_{1}}^{-}, v_{\alpha_{2}}^{-}\right)+v_{\alpha_{3}}^{-}\right.}, \frac{2 \min \left(v_{\alpha_{1}}^{+}, v_{\alpha_{2}}^{+}\right) v_{\alpha_{3}}^{+}}{\min \left(v_{\alpha_{1}}^{+}, v_{\alpha_{2}}^{+}\right)+v_{\alpha_{3}}^{+}}\right] \mid \alpha_{1} \in \tilde{h}_{1}, \alpha_{2} \in \tilde{h}_{2}, \alpha_{3} \in \tilde{h}_{3}\right\} \\
=\left\{\left[\max \left(\frac{2 \mu_{\alpha_{1}} \mu_{\alpha_{3}}^{-}}{\mu_{\alpha_{1}}+\mu_{\alpha_{3}}^{-}}, \frac{2 \mu_{\alpha_{2}}^{-} \mu_{\alpha_{3}}^{-}}{\mu_{\overline{\alpha_{2}}}+\mu_{\alpha_{3}}^{-}}\right), \max \left(\frac{2 \mu_{\alpha_{1}}^{+} \mu_{\alpha_{3}}^{+}}{\mu_{\alpha_{1}}^{+}+\mu_{\alpha_{3}}^{+}}, \frac{2 \mu_{\alpha_{2}}^{+} \mu_{\alpha_{3}}^{+}}{\mu_{\alpha_{2}}^{+}+\mu_{\alpha_{3}}^{+}}\right)\right],\left[\min \left(\frac{2 v_{\alpha_{1}}^{-} v_{\alpha_{3}}^{-}}{v_{\alpha_{1}}+v_{\alpha_{3}}^{-}}, \frac{2 v_{\alpha_{2}}^{-} v_{\alpha_{3}}^{-}}{v_{\alpha_{2}}+v_{\alpha_{3}}^{-}}\right), \min \left(\frac{2 v_{\alpha_{1}}^{+} v_{\alpha_{3}}^{+}}{v_{\alpha_{1}}^{+}+v_{\alpha_{3}}^{+}},\right.\right.\right. \\
\left.\left.\left.\frac{2 v_{\alpha_{2}}^{+} v_{\alpha_{3}}^{+}}{v_{\alpha_{2}}^{+}+v_{\alpha_{3}}^{+}}\right)\right] \mid \alpha_{1} \in \tilde{h}_{1}, \alpha_{2} \in \tilde{h}_{2}, \alpha_{3} \in \tilde{h}_{3}\right\}
\end{gathered}
$$

$=\left(\tilde{h}_{1} \# \tilde{h}_{3}\right) \cup\left(\tilde{h}_{2} \# \tilde{h}_{3}\right)$

This proves (v)

(vii) From definitions 2.3 and 3.1, we have

$\left(\tilde{h}_{1} \cup \tilde{h}_{2}\right) * \tilde{h}_{3}=\left(\tilde{h}_{1} * \tilde{h}_{3}\right) \cup\left(\tilde{h}_{2} * \tilde{h}_{3}\right)$

$=\left\{\left[\max \left(\mu_{\alpha_{1}}^{-}, \mu_{\alpha_{2}}^{-}\right), \max \left(\mu_{\alpha_{1}}^{+}, \mu_{\alpha_{2}}^{+}\right)\right],\left[\min :\left(v_{\alpha_{1}}^{-}, v_{\alpha_{2}}^{-}\right), \min \left(v_{\alpha_{1}}^{+}, v_{\alpha_{2}}^{+}\right)\right] \mid \alpha_{1} \in \tilde{h}_{1}, \alpha_{2} \in \tilde{h}_{2}\right\} *\left\{\left[\mu_{\alpha_{3}}^{-}, \mu_{\alpha_{3}}^{+}\right],\left[v_{\alpha_{3}}^{-}\right.\right.$,

$\left.\left.v_{\alpha_{3}}^{+}\right] \mid \alpha_{3} \in \widetilde{h}_{3}\right\}$

$$
\begin{gathered}
=\left\{\left[\frac{\max \left(\mu_{\alpha_{1}}^{-}, \mu_{\alpha_{2}}^{-}\right)+\mu_{\alpha_{3}}^{-}}{2\left(\max \left(\mu_{\alpha_{1}}^{-}, \mu_{\alpha_{2}}^{-}\right) \mu_{\alpha_{3}}^{-}+1\right)}, \frac{\max \left(\mu_{\alpha_{1}}^{+}, \mu_{\alpha_{2}}^{+}\right)+\mu_{\alpha_{3}}^{+}}{\max \left(\mu_{\alpha_{1}}^{+}, \mu_{\alpha_{2}}^{+}\right) \mu_{\alpha_{2}}^{+}+1}\right],\left[\frac{\min \left(v_{\alpha_{1}}^{-}, v_{\alpha_{2}}^{-}\right)+v_{\alpha_{3}}^{-}}{2\left(\min \left(v_{\alpha_{1}}^{-}, v_{\alpha_{2}}^{-}\right) v_{\alpha_{3}}^{-}+1\right)}, \frac{\min \left(v_{\alpha_{1}}^{+}, v_{\alpha_{2}}^{+}\right)+v_{\alpha_{3}}^{+}}{2\left(\min \left(v_{\alpha_{1}}^{+}, v_{\alpha_{2}}^{+}\right) v_{\alpha_{3}}^{+}+1\right)}\right] \mid \alpha_{1} \in \tilde{h}_{1}, \alpha_{2} \in \tilde{h}_{2}, \alpha_{3} \in \tilde{h}_{3}\right\} \\
=\left\{\left[\max \left(\frac{\mu_{\alpha_{1}}^{-}+\mu_{\alpha_{3}}^{-}}{2\left(\mu_{\alpha_{1}}^{-} \mu_{\alpha_{3}}^{-}+1\right)}, \frac{\mu_{\alpha_{2}}^{-}+\mu_{\alpha_{3}}^{-}}{2\left(\mu_{\alpha_{2}}^{-} \mu_{\alpha_{3}}^{-}+1\right)}\right), \max \left(\frac{\mu_{\alpha_{1}+}^{+} \mu_{\alpha_{3}}^{+}}{2\left(\mu_{\alpha_{1}}^{+} \mu_{\alpha_{3}}^{+}+1\right)}, \frac{\mu_{\alpha_{2}}^{+}+\mu_{\alpha_{3}}^{+}}{2\left(\mu_{\alpha_{2}}^{+} \mu_{\alpha_{3}}^{+}+1\right)}\right)\right],\left[\min \left(\frac{v_{\alpha_{1}}^{-}+v_{\alpha_{3}}^{-}}{2\left(v_{\alpha_{1}}^{-} v_{\alpha_{3}}^{-}+1\right)}, \frac{v_{\alpha_{2}}^{-}+v_{\alpha_{3}}^{-}}{2\left(v_{\alpha_{2}}^{-} v_{\alpha_{3}}^{-}+1\right)}\right), \min \right.\right. \\
\\
\left.\left.\left(\frac{v_{\alpha_{1}}^{+}+v_{\alpha_{3}}^{+}}{2\left(v_{\alpha_{1}}^{+} v_{\alpha_{3}}^{+}+1\right)}, \frac{v_{\alpha_{2}}^{+}+v_{\alpha_{3}}^{+}}{2\left(v_{\alpha_{2}}^{+} v_{\alpha_{3}}^{+}+1\right)}\right)\right] \mid \alpha_{1} \in \tilde{h}_{1}, \alpha_{2} \in \tilde{h}_{2}, \alpha_{3} \in \tilde{h}_{3}\right\}
\end{gathered}
$$

$=\left(\tilde{h}_{1} * \tilde{h}_{3}\right) \cup\left(\tilde{h}_{2} * \tilde{h}_{3}\right)$

This proves (vii).

(ix) Using definitions 2.3 and 3.1, we have

$\left(\tilde{h}_{1} @ \widetilde{h}_{2}\right) \oplus \tilde{h}_{3}=\left(\tilde{h}_{1} \oplus \tilde{h}_{3}\right) @\left(\tilde{h}_{2} \oplus \tilde{h}_{3}\right) ;$

$=\left\{\left[\frac{\mu_{\alpha_{1}}+\mu_{\alpha_{2}}^{-}}{2}, \frac{\mu_{\alpha_{1}}^{+}+\mu_{\alpha_{2}}^{+}}{2}\right],\left[\frac{v_{\alpha_{1}}+v_{\alpha_{2}}^{-}}{2}, \frac{v_{\alpha_{1}}^{+}+v_{\alpha_{2}}^{+}}{2}\right] \mid \alpha_{1} \in \tilde{h}_{1}, \alpha_{2} \in \tilde{h}_{2}\right\} \oplus\left\{\left[\mu_{\alpha_{3}}^{-}, \mu_{\alpha_{3}}^{+}\right],\left[v_{\alpha_{3}}^{-}, v_{\alpha_{3}}^{+}\right] \mid \alpha_{3} \in \tilde{h}_{3}\right\}$

$=\left\{\left[\frac{\mu_{\alpha_{1}}+\mu_{\alpha_{2}}^{-}}{2}+\mu_{\alpha_{3}}^{-}-\frac{\mu_{\alpha_{1}}+\mu_{\alpha_{2}}^{-}}{2} \mu_{\alpha_{3}}^{-}, \frac{\mu_{\alpha_{1}}^{+}+\mu_{\alpha_{2}}^{+}}{2}+\mu_{\alpha_{3}}^{+}-\frac{\mu_{\alpha_{1}}^{+}+\mu_{\alpha_{2}}^{+}}{2} \mu_{\alpha_{3}}^{+}\right],\left[\frac{v_{\alpha_{1}}^{-}+v_{\alpha_{2}}^{-}}{2}+v_{\alpha_{3}}^{-}-\frac{v_{\alpha_{1}}^{-}+v_{\alpha_{2}}^{-}}{2} v_{\alpha_{3}}^{-}, \frac{v_{\alpha_{1}}^{+}+v_{\alpha_{2}}^{+}}{2}+v_{\alpha_{3}}^{+}\right.\right.$

$\left.\left.-\frac{v_{\alpha_{1}+v_{\alpha_{2}}^{+}}^{+}}{2} v_{\alpha_{3}}^{+}\right] \mid \alpha_{1} \in \tilde{h}_{1}, \alpha_{2} \in \tilde{h}_{2}, \alpha_{3} \in \tilde{h}_{3}\right\}$

$=\left\{\left[\frac{\left(\mu_{\alpha_{1}}+\mu_{\alpha_{3}}-\mu_{\alpha_{1}} \mu_{\alpha_{3}}\right)+\left(\mu_{\alpha_{2}}+\mu_{\alpha_{3}}-\mu_{\alpha_{2}} \mu_{\alpha_{3}}\right)}{2}, \frac{\left(\mu_{\alpha_{1}}^{+}+\mu_{\alpha_{3}}^{+}-\mu_{\alpha_{1}}^{+} \mu_{\alpha_{3}}^{+}\right)+\left(\mu_{\alpha_{2}}^{+}+\mu_{\alpha_{3}}^{+}-\mu_{\alpha_{2}}^{+} \mu_{\alpha_{3}}^{+}\right)}{2}\right],\left[\frac{\left(v_{\alpha_{1}}+v_{\alpha_{3}}^{-}-v_{\alpha_{1}} v_{\alpha_{3}}^{-}\right)+\left(v_{\alpha_{2}}+v_{\alpha_{3}}-v_{\alpha_{2}}^{-} v_{\alpha_{3}}\right)}{2}\right.\right.$,

$\left.\left.\frac{\left(v_{\alpha_{1}}^{+}+v_{\alpha_{3}}^{+}-v_{\alpha_{1}}^{+} v_{\alpha_{3}}^{+}\right)+\left(v_{\alpha_{2}}^{+}+v_{\alpha_{3}}^{+}-v_{\alpha_{2}}^{+} v_{\alpha_{3}}^{+}\right)}{2}\right] \mid \alpha_{1} \in \tilde{h}_{1}, \alpha_{2} \in \tilde{h}_{2}, \alpha_{3} \in \tilde{h}_{3}\right\}$

$=\left(\tilde{h}_{1} \oplus \tilde{h}_{3}\right) @\left(\tilde{h}_{2} \oplus \tilde{h}_{3}\right)$

This proves (ix)

\section{Theorem 3.7}

For $\tilde{h}_{1}$ and $\tilde{h}_{2} \in$ IVIHFS (X), we have the following identities:

(i) $\quad\left(\tilde{h}_{1} \oplus \widetilde{h}_{2}\right) \cap\left(\tilde{h}_{1} \otimes \tilde{h}_{2}\right)=\tilde{h}_{1} \otimes \tilde{h}_{2} ;$ 
(ii) $\quad\left(\tilde{h}_{1} \oplus \tilde{h}_{2}\right) \cup\left(\tilde{h}_{1} \otimes \tilde{h}_{2}\right)=\tilde{h}_{1} \oplus \tilde{h}_{2}$;

(iii) $\quad\left(\tilde{h}_{1} \oplus \widetilde{h}_{2}\right) \cap\left(\tilde{h}_{1} @ \widetilde{h}_{2}\right)=\tilde{h}_{1} @ \widetilde{h}_{2}$;

(iv) $\quad\left(\tilde{h}_{1} \oplus \widetilde{h}_{2}\right) \cup\left(\widetilde{h}_{1} @ \widetilde{h}_{2}\right)=\widetilde{h}_{1} \oplus \widetilde{h}_{2}$;

(v) $\quad\left(\tilde{h}_{1} \otimes \tilde{h}_{2}\right) \cap\left(\tilde{h}_{1} @ \tilde{h}_{2}\right)=\tilde{h}_{1} \otimes \tilde{h}_{2}$;

(vi) $\quad\left(\tilde{h}_{1} \otimes \tilde{h}_{2}\right) \cup\left(\tilde{h}_{1} @ \tilde{h}_{2}\right)=\tilde{h}_{1} @ \tilde{h}_{2}$;

(vii) $\quad\left(\tilde{h}_{1} \oplus \tilde{h}_{2}\right) \cap\left(\tilde{h}_{1} \$ \widetilde{h}_{2}\right)=\widetilde{h}_{1} \$ \widetilde{h}_{2}$;

(viii) $\quad\left(\tilde{h}_{1} \oplus \tilde{h}_{2}\right) \cup\left(\tilde{h}_{1} \$ \widetilde{h}_{2}\right)=\tilde{h}_{1} \oplus \widetilde{h}_{2}$;

(ix) $\quad\left(\tilde{h}_{1} \otimes \tilde{h}_{2}\right) \cap\left(\tilde{h}_{1} \$ \widetilde{h}_{2}\right)=\tilde{h}_{1} \otimes \tilde{h}_{2}$;

(x) $\quad\left(\tilde{h}_{1} \otimes \tilde{h}_{2}\right) \cup\left(\tilde{h}_{1} \$ \tilde{h}_{2}\right)=\tilde{h}_{1} \$ \tilde{h}_{2}$;

(xi) $\quad\left(\tilde{h}_{1} \oplus \widetilde{h}_{2}\right) \cap\left(\tilde{h}_{1} \# \tilde{h}_{2}\right)=\tilde{h}_{1} \# \tilde{h}_{2}$;

(xii) $\quad\left(\tilde{h}_{1} \oplus \widetilde{h}_{2}\right) \cup\left(\tilde{h}_{1} \# \tilde{h}_{2}\right)=\tilde{h}_{1} \oplus \tilde{h}_{2}$;

(xiii) $\quad\left(\tilde{h}_{1} \otimes \tilde{h}_{2}\right) \cap\left(\tilde{h}_{1} \# \tilde{h}_{2}\right)=\tilde{h}_{1} \otimes \tilde{h}_{2}$;

(xiv) $\quad\left(\tilde{h}_{1} \otimes \tilde{h}_{2}\right) \cup\left(\tilde{h}_{1} \# \tilde{h}_{2}\right)=\tilde{h}_{1} \# \tilde{h}_{2}$

Proof. We prove (i), (iii), (v), (vii), (ix), (xi) and (xii), other results can be proved analogously.

From definitions 2.3, 2.5 and 3.1, we have

$\left(\tilde{h}_{1} \oplus \tilde{h}_{2}\right) \cap\left(\tilde{h}_{1} \otimes \tilde{h}_{2}\right)$

$$
\begin{aligned}
& =\left(\left[\mu_{\alpha_{2}}^{-}+\mu_{\alpha_{1}}^{-}-\mu_{\alpha_{2}}^{-} \mu_{\alpha_{1}}^{-}, \mu_{\alpha_{2}}^{+}+\mu_{\alpha_{1}}^{+}-\mu_{\alpha_{2}}^{+} \mu_{\alpha_{1}}^{+}\right],\left[v_{\alpha_{2}}^{-} v_{\alpha_{1}}^{-}, v_{\alpha_{2}}^{+} v_{\alpha_{1}}^{+}\right]\right) \cap\left\{\left[\mu_{\alpha_{1}}^{-} \mu_{\alpha_{2}}^{-}, \mu_{\alpha_{1}}^{+} \mu_{\alpha_{2}}^{+}\right],\left[v_{\alpha_{1}}^{-}+v_{\alpha_{2}}^{-}-v_{\alpha_{1}}^{-} v_{\alpha_{2}}^{-}, v_{\alpha_{1}}^{+}+\right.\right. \\
& \left.\left.v_{\alpha_{2}}^{+}-v_{\alpha_{1}}^{+} v_{\alpha_{2}}^{+}\right] \mid \alpha_{1} \in \tilde{h}_{1}, \alpha_{2} \in \tilde{h}_{2}\right\} \\
= & \left\{\left[\min \left(\mu_{\alpha_{2}}^{-}+\mu_{\alpha_{1}}^{-}-\mu_{\alpha_{2}}^{-} \mu_{\alpha_{1}}^{-}, \mu_{\alpha_{1}}^{-} \mu_{\alpha_{2}}^{-}\right), \min \left(\mu_{\alpha_{2}}^{+}+\mu_{\alpha_{1}}^{+}-\mu_{\alpha_{2}}^{+} \mu_{\alpha_{1}}^{+}, \mu_{\alpha_{1}}^{+} \mu_{\alpha_{2}}^{+}\right)\right],\right. \\
& {\left.\left[\max \left(v_{\alpha_{2}}^{-} v_{\alpha_{1}}^{-}, v_{\alpha_{1}}^{-}+v_{\alpha_{2}}^{-}-v_{\alpha_{1}}^{-} v_{\alpha_{2}}^{-}\right), \max \left(v_{\alpha_{2}}^{+} v_{\alpha_{1}}^{+}, v_{\alpha_{1}}^{+}+v_{\alpha_{2}}^{+}-v_{\alpha_{1}}^{+} v_{\alpha_{2}}^{+}\right)\right] \mid \alpha_{1} \in \tilde{h}_{1}, \alpha_{2} \in \tilde{h}_{2}\right\} } \\
= & {\left.\left.\left[\mu_{\alpha_{1}}^{-} \mu_{\alpha_{2}}^{-}, \mu_{\alpha_{1}}^{+} \mu_{\alpha_{2}}^{+}\right)\right],\left[v_{\alpha_{1}}^{-}+v_{\alpha_{2}}^{-}-v_{\alpha_{1}}^{-} v_{\alpha_{2}}^{-}, v_{\alpha_{1}}^{+}+v_{\alpha_{2}}^{+}-v_{\alpha_{1}}^{+} v_{\alpha_{2}}^{+}\right] \mid \alpha_{1} \in \tilde{h}_{1}, \alpha_{2} \in \tilde{h}_{2}\right\} } \\
= & \tilde{h}_{1} \otimes \tilde{h}_{2}
\end{aligned}
$$

This proves (i)

iii) Using definitions 2.3, 2.5 and 3.1, we have

$\left(\tilde{h}_{1} \oplus \tilde{h}_{2}\right) \cap\left(\tilde{h}_{1} @ \tilde{h}_{2}\right)=\tilde{h}_{1} @ \tilde{h}_{2} ;$

$=\left\{\left(\left[\mu_{\alpha_{2}}^{-}+\mu_{\alpha_{1}}^{-}-\mu_{\alpha_{2}}^{-} \mu_{\alpha_{1}}^{-}, \mu_{\alpha_{2}}^{+}+\mu_{\alpha_{1}}^{+}-\mu_{\alpha_{2}}^{+} \mu_{\alpha_{1}}^{+}\right],\left[v_{\alpha_{2}}^{-} v_{\alpha_{1}}^{-}, v_{\alpha_{2}}^{+} v_{\alpha_{1}}^{+}\right]\right) \mid \alpha_{1} \in \tilde{h}_{1}, \alpha_{2} \in \tilde{h}_{2}\right\} \cap\left\{\left[\frac{\mu_{\alpha_{1}}+\mu_{\alpha_{2}}}{2}\right.\right.$,

$\left.\left.\frac{\mu_{\alpha_{1}}^{+}+\mu_{\alpha_{2}}^{+}}{2}\right],\left[\frac{v_{\alpha_{1}}+v_{\alpha_{2}}^{-}}{2}, \frac{v_{\alpha_{1}}^{+}+v_{\alpha_{2}}^{+}}{2}\right] \mid \alpha_{1} \in \tilde{h}_{1}, \alpha_{2} \in \tilde{h}_{2}\right\}$

$=\left\{\left[\min \left(\mu_{\alpha_{2}}^{-}+\mu_{\alpha_{1}}^{-}-\mu_{\alpha_{2}}^{-} \mu_{\alpha_{1}}^{-}, \frac{\mu_{\alpha_{1}}+\mu_{\alpha_{2}}^{-}}{2}\right), \min \left(\mu_{\alpha_{1}}^{-}, \mu_{\alpha_{2}}^{+}+\mu_{\alpha_{1}}^{+}-\mu_{\alpha_{2}}^{+} \mu_{\alpha_{1}}^{+}, \frac{\mu_{\alpha_{1}}^{+}+\mu_{\alpha_{2}}^{+}}{2}\right)\right]\right.$,

$\left.\left[\max \left(v_{\alpha_{2}}^{-} v_{\alpha_{1}}^{-}, \frac{v_{\alpha_{1}}^{-}+v_{\alpha_{2}}^{-}}{2}\right), \max \left(v_{\alpha_{2}}^{+} v_{\alpha_{1}}^{+}, \frac{v_{\alpha_{1}}^{+}+v_{\alpha_{2}}^{+}}{2}\right)\right] \mid \alpha_{1} \in \tilde{h}_{1}, \alpha_{2} \in \tilde{h}_{2}\right\}$

$=\left\{\left[\frac{\mu_{\alpha_{1}}^{-}+\mu_{\alpha_{2}}^{-}}{2}, \frac{\mu_{\alpha_{1}}^{+}+\mu_{\alpha_{2}}^{+}}{2}\right],\left[\frac{v_{\alpha_{1}}^{-}+v_{\alpha_{2}}^{-}}{2}, \frac{v_{\alpha_{1}}^{+}+v_{\alpha_{2}}^{+}}{2}\right] \mid \alpha_{1} \in \tilde{h}_{1}, \alpha_{2} \in \tilde{h}_{2}\right\}=\tilde{h}_{1} @ \tilde{h}_{2}$

This proves (iii).

(v) From definitions 2.3, 2.5 and 3.1, we have

$\left(\tilde{h}_{1} \otimes \tilde{h}_{2}\right) \cap\left(\tilde{h}_{1} @ \tilde{h}_{2}\right)=\tilde{h}_{1} \otimes \tilde{h}_{2} ;$

$=\left\{\left[\mu_{\alpha_{1}}^{-} \mu_{\alpha_{2}}^{-}, \mu_{\alpha_{1}}^{+} \mu_{\alpha_{2}}^{+}\right],\left[v_{\alpha_{1}}^{-}+v_{\alpha_{2}}^{-}-v_{\alpha_{1}}^{-} v_{\alpha_{2}}^{-}, v_{\alpha_{1}}^{+}+v_{\alpha_{2}}^{+}-v_{\alpha_{1}}^{+} v_{\alpha_{2}}^{+}\right] \mid \alpha_{1} \in \tilde{h}_{1}, \alpha_{2} \in \tilde{h}_{2}\right\} \cap\left\{\left[\frac{\mu_{\alpha_{1}}^{-}+\mu_{\alpha_{2}}^{-}}{2}, \frac{\mu_{\alpha_{1}}^{+}+\mu_{\alpha_{2}}^{+}}{2}\right]\right.$,

$\left.\left[\frac{v_{\alpha_{1}}+v_{\alpha_{2}}^{-}}{2}, \frac{v_{\alpha_{1}}^{+}+v_{\alpha_{2}}^{+}}{2}\right] \mid \alpha_{1} \in \tilde{h}_{1}, \alpha_{2} \in \tilde{h}_{2}\right\}$

$=\left\{\left[\min \left(\mu_{\alpha_{1}}^{-} \mu_{\alpha_{2}}^{-}, \frac{\mu_{\alpha_{1}}+\mu_{\alpha_{2}}^{-}}{2}\right), \min \left(\mu_{\alpha_{1}}^{+} \mu_{\alpha_{2}}^{+}, \frac{\mu_{\alpha_{1}+\mu_{\alpha_{2}}^{+}}^{+}}{2}\right)\right]\right.$,

$\left.\left[\max \left(v_{\alpha_{1}}^{-}+v_{\alpha_{2}}^{-}-v_{\alpha_{1}}^{-} v_{\alpha_{2}}^{-}, \frac{v_{\alpha_{1}}+v_{\alpha_{2}}^{-}}{2}\right), \max \left(v_{\alpha_{1}}^{+}+v_{\alpha_{2}}^{+}-v_{\alpha_{1}}^{+} v_{\alpha_{2}}^{+}, \frac{v_{\alpha_{1}}^{+}+v_{\alpha_{2}}^{+}}{2}\right)\right] \mid \alpha_{1} \in \tilde{h}_{1}, \alpha_{2} \in \tilde{h}_{2}\right\}$

$=\left\{\left[\mu_{\alpha_{1}}^{-} \mu_{\alpha_{2}}^{-}, \mu_{\alpha_{1}}^{+} \mu_{\alpha_{2}}^{+}\right],\left[v_{\alpha_{1}}^{-}+v_{\alpha_{2}}^{-}-v_{\alpha_{1}}^{-} v_{\alpha_{2}}^{-}, v_{\alpha_{1}}^{+}+v_{\alpha_{2}}^{+}-v_{\alpha_{1}}^{+} v_{\alpha_{2}}^{+}\right] \mid \alpha_{1} \in \tilde{h}_{1}, \alpha_{2} \in \tilde{h}_{2}\right\}=\tilde{h}_{1} \otimes \tilde{h}_{2}$

This proves (v)

(vii) Using definitions 2.3, 2.5 and 3.1, we have

$\left(\tilde{h}_{1} \oplus \tilde{h}_{2}\right) \cap\left(\tilde{h}_{1} \$ \tilde{h}_{2}\right)=\tilde{h}_{1} \$ \tilde{h}_{2}$ 
$=\left(\left[\mu_{\alpha_{2}}^{-}+\mu_{\alpha_{1}}^{-}-\mu_{\alpha_{2}}^{-} \mu_{\alpha_{1}}^{-}, \mu_{\alpha_{2}}^{+}+\mu_{\alpha_{1}}^{+}-\mu_{\alpha_{2}}^{+} \mu_{\alpha_{1}}^{+}\right],\left[v_{\alpha_{2}}^{-} v_{\alpha_{1}}^{-}, v_{\alpha_{2}}^{+} v_{\alpha_{1}}^{+}\right]\right) \cap\left\{\left[\sqrt{\mu_{\alpha_{1}}^{-} \mu_{\alpha_{2}}^{-}}, \sqrt{\mu_{\alpha_{1}}^{+} \mu_{\alpha_{2}}^{+}}\right],\left[\sqrt{v_{\alpha_{1}}^{-} v_{\alpha_{2}}^{-}}\right.\right.$,

$\left.\left.\sqrt{v_{\alpha_{1}}^{+} v_{\alpha_{2}}^{+}}\right] \mid \alpha_{1} \in \tilde{h}_{1}, \alpha_{2} \in \tilde{h}_{2}\right\}$

$=\left\{\left[\min \left(\mu_{\alpha_{2}}^{-}+\mu_{\alpha_{1}}^{-}-\mu_{\alpha_{2}}^{-} \mu_{\alpha_{1}}^{-}, \sqrt{\mu_{\alpha_{1}}^{-} \mu_{\alpha_{2}}^{-}}\right), \min \left(\mu_{\alpha_{2}}^{+}+\mu_{\alpha_{1}}^{+}-\mu_{\alpha_{2}}^{+} \mu_{\alpha_{1}}^{+}, \sqrt{\mu_{\alpha_{1}}^{+} \mu_{\alpha_{2}}^{+}}\right)\right],\left[\max \left(v_{\alpha_{2}}^{-} v_{\alpha_{1}}^{-}, \sqrt{v_{\alpha_{1}}^{-} v_{\alpha_{2}}^{-}}\right), \max \right.\right.$ $\left.\left.\left(v_{\alpha_{2}}^{+} v_{\alpha_{1}}^{+}, \sqrt{v_{\alpha_{1}}^{+} v_{\alpha_{2}}^{+}}\right)\right] \mid \alpha_{1} \in \tilde{h}_{1}, \alpha_{2} \in \tilde{h}_{2}\right\}$

$=\left\{\left[\sqrt{\mu_{\alpha_{1}}^{-} \mu_{\alpha_{2}}^{-}}, \sqrt{\mu_{\alpha_{1}}^{+} \mu_{\alpha_{2}}^{+}}\right],\left[\sqrt{v_{\alpha_{1}}^{-} v_{\alpha_{2}}^{-}}, \sqrt{v_{\alpha_{1}}^{+} v_{\alpha_{2}}^{+}}\right] \mid \alpha_{1} \in \tilde{h}_{1}, \alpha_{2} \in \tilde{h}_{2}\right\}=\tilde{h}_{1} \$ \tilde{h}_{2}$

This proves (vii)

(ix) From definitions 2.3, 2.5 and 3.1, we have

$\left(\tilde{h}_{1} \otimes \tilde{h}_{2}\right) \cap\left(\tilde{h}_{1} \$ \tilde{h}_{2}\right)=\tilde{h}_{1} \otimes \tilde{h}_{2} ;$

$=\left\{\left(\left[\mu_{\alpha_{1}}^{-} \mu_{\alpha_{2}}^{-}, \mu_{\alpha_{1}}^{+} \mu_{\alpha_{2}}^{+}\right],\left[v_{\alpha_{1}}^{-}+v_{\alpha_{2}}^{-}-v_{\alpha_{1}}^{-} v_{\alpha_{2}}^{-}, v_{\alpha_{1}}^{+}+v_{\alpha_{2}}^{+}-v_{\alpha_{1}}^{+} v_{\alpha_{2}}^{+}\right]\right) \mid \alpha_{1} \in \tilde{h}_{1}, \alpha_{2} \in \tilde{h}_{2}\right\} \cap\left\{\left[\sqrt{\mu_{\alpha_{1}}^{-} \mu_{\alpha_{2}}^{-}}\right.\right.$,

$\left.\left.\sqrt{\mu_{\alpha_{1}}^{+} \mu_{\alpha_{2}}^{+}}\right],\left[\sqrt{v_{\alpha_{1}}^{-} v_{\alpha_{2}}^{-}}, \sqrt{v_{\alpha_{1}}^{+} v_{\alpha_{2}}^{+}}\right] \mid \alpha_{1} \in \tilde{h}_{1}, \alpha_{2} \in \tilde{h}_{2}\right\}$

$=\left\{\left[\min \left(\mu_{\alpha_{1}}^{-} \mu_{\alpha_{2}}^{-}, \sqrt{\mu_{\alpha_{1}}^{-} \mu_{\alpha_{2}}^{-}}\right), \min \left(\mu_{\alpha_{1}}^{+} \mu_{\alpha_{2}}^{+}, \sqrt{\mu_{\alpha_{1}}^{+} \mu_{\alpha_{2}}^{+}}\right)\right],\left[\max \left(v_{\alpha_{1}}^{-}+v_{\alpha_{2}}^{-}-v_{\alpha_{1}}^{-} v_{\alpha_{2}}^{-}, \sqrt{v_{\alpha_{1}}^{-} v_{\alpha_{2}}^{-}}\right), \max \right.\right.$

$\left.\left.\left(v_{\alpha_{1}}^{+}+v_{\alpha_{2}}^{+}-v_{\alpha_{1}}^{+} v_{\alpha_{2}}^{+}, \sqrt{v_{\alpha_{1}}^{+} v_{\alpha_{2}}^{+}}\right)\right] \mid \alpha_{1} \in \tilde{h}_{1}, \alpha_{2} \in \tilde{h}_{2}\right\}$

$=\left\{\left[\mu_{\alpha_{1}}^{-} \mu_{\alpha_{2}}^{-}, \mu_{\alpha_{1}}^{+} \mu_{\alpha_{2}}^{+}\right],\left[v_{\alpha_{1}}^{-}+v_{\alpha_{2}}^{-}-v_{\alpha_{1}}^{-} v_{\alpha_{2}}^{-}, v_{\alpha_{1}}^{+}+v_{\alpha_{2}}^{+}-v_{\alpha_{1}}^{+} v_{\alpha_{2}}^{+}\right] \mid \alpha_{1} \in \tilde{h}_{1}, \alpha_{2} \in \tilde{h}_{2}\right\}=\tilde{h}_{1} \otimes \tilde{h}_{2}$

This proves (ix)

(xiii) From definitions 2.3, 2.5 and 3.1, we have

$\left(\tilde{h}_{1} \otimes \tilde{h}_{2}\right) \cap\left(\tilde{h}_{1} \# \widetilde{h}_{2}\right)=\tilde{h}_{1} \otimes \tilde{h}_{2} ;$

$=\left\{\left[\mu_{\alpha_{1}}^{-} \mu_{\alpha_{2}}^{-}, \mu_{\alpha_{1}}^{+} \mu_{\alpha_{2}}^{+}\right],\left[v_{\alpha_{1}}^{-}+v_{\alpha_{2}}^{-}-v_{\alpha_{1}}^{-} v_{\alpha_{2}}^{-}, v_{\alpha_{1}}^{+}+v_{\alpha_{2}}^{+}-v_{\alpha_{1}}^{+} v_{\alpha_{2}}^{+}\right] \mid \alpha_{1} \in \tilde{h}_{1}, \alpha_{2} \in \tilde{h}_{2}\right\} \cap\left\{\left[\frac{2 \mu_{\alpha_{1}}^{-} \mu_{\alpha_{2}}^{-}}{\mu_{\alpha_{1}}+\mu_{\alpha_{2}}^{-}}\right.\right.$,

$\left.\left.\frac{2 \mu_{\alpha_{1}}^{+} \mu_{\alpha_{2}}^{+}}{\mu_{\alpha_{1}}^{+}+\mu_{\alpha_{2}}^{+}}\right],\left[\frac{2 v_{\alpha_{1}}^{-} v_{\alpha_{2}}^{-}}{v_{\alpha_{1}}^{-}+v_{\alpha_{2}}^{-}}, \frac{2 v_{\alpha_{1}}^{+} v_{\alpha_{2}}^{+}}{v_{\alpha_{1}}^{+}+v_{\alpha_{2}}^{+}}\right] \mid \alpha_{1} \in \tilde{h}_{1}, \alpha_{2} \in \tilde{h}_{2}\right\}$

$=\left\{\left[\min \left(\mu_{\alpha_{1}}^{-} \mu_{\alpha_{2}}^{-}, \frac{2 \mu_{\alpha_{1}}^{-} \mu_{\alpha_{2}}^{-}}{\mu_{\alpha_{1}}^{-}+\mu_{\alpha_{2}}^{-}}\right), \min \left(\mu_{\alpha_{1}}^{+} \mu_{\alpha_{2}}^{+}, \frac{2 \mu_{\alpha_{1}}^{+} \mu_{\alpha_{2}}^{+}}{\mu_{\alpha_{1}}^{+}+\mu_{\alpha_{2}}^{+}}\right)\right],\left[\max \left(v_{\alpha_{1}}^{-}+v_{\alpha_{2}}^{-}-v_{\alpha_{1}}^{-} v_{\alpha_{2}}^{-}, \frac{2 v_{\alpha_{1}}^{-} v_{\alpha_{2}}^{-}}{v_{\alpha_{1}}^{-}+v_{\alpha_{2}}^{-}}\right), \max \left(v_{\alpha_{1}}^{+}+v_{\alpha_{2}}^{+}-\right.\right.\right.$ $\left.\left.v_{\alpha_{1}}^{+} v_{\alpha_{2}}^{+}, \frac{2 v_{\alpha_{1}}^{+} v_{\alpha_{2}}^{+}}{v_{\alpha_{1}}^{+}+v_{\alpha_{2}}^{+}}\right] \mid \alpha_{1} \in \tilde{h}_{1}, \alpha_{2} \in \tilde{h}_{2}\right\}$

$=\left\{\left[\mu_{\alpha_{1}}^{-} \mu_{\alpha_{2}}^{-}, \mu_{\alpha_{1}}^{+} \mu_{\alpha_{2}}^{+}\right],\left[v_{\alpha_{1}}^{-}+v_{\alpha_{2}}^{-}-v_{\alpha_{1}}^{-} v_{\alpha_{2}}^{-}, v_{\alpha_{1}}^{+}+v_{\alpha_{2}}^{+}-v_{\alpha_{1}}^{+} v_{\alpha_{2}}^{+}\right] \mid \alpha_{1} \in \tilde{h}_{1}, \alpha_{2} \in \tilde{h}_{2}\right\}=\tilde{h}_{1} \otimes \tilde{h}_{2}$

This proves (xiii).

\section{Theorem 3.8}

For $\tilde{h}_{1}$ and $\tilde{h}_{2} \in \operatorname{IVIHFE}(\mathrm{X})$, then following relations are valid:

(i) $\quad\left(\tilde{h}_{1} \# \tilde{h}_{2}\right) \$\left(\tilde{h}_{1} \# \tilde{h}_{2}\right)=\tilde{h}_{1} \# \tilde{h}_{2}$;

(ii) $\quad\left(\tilde{h}_{1} * \tilde{h}_{2}\right) \$\left(\tilde{h}_{1} * \tilde{h}_{2}\right)=\tilde{h}_{1} * \tilde{h}_{2}$;

(iii) $\quad\left(\tilde{h}_{1} \oplus \tilde{h}_{2}\right) \$\left(\tilde{h}_{1} \oplus \quad \tilde{h}_{2}\right)=\tilde{h}_{1} \oplus \tilde{h}_{2}$;

(iv) $\quad\left(\tilde{h}_{1} \otimes \tilde{h}_{2}\right) \$\left(\tilde{h}_{1} \otimes \tilde{h}_{2}\right)=\tilde{h}_{1} \otimes \tilde{h}_{2}$;

(v) $\quad\left(\tilde{h}_{1} @ \widetilde{h}_{2}\right) \$\left(\tilde{h}_{1} @ \widetilde{h}_{2}\right)=\tilde{h}_{1} @ \tilde{h}_{2}$;

(vi) $\quad\left(\tilde{h}_{1} \# \widetilde{h}_{2}\right) @\left(\tilde{h}_{1} \# \widetilde{h}_{2}\right)=\tilde{h}_{1} \# \widetilde{h}_{2}$;

(vii) $\left(\tilde{h}_{1} * \widetilde{h}_{2}\right) @\left(\tilde{h}_{1} * \tilde{h}_{2}\right)=\tilde{h}_{1} * \tilde{h}_{2}$;

(viii) $\quad\left(\tilde{h}_{1} \oplus \tilde{h}_{2}\right) @\left(\tilde{h}_{1} \otimes \tilde{h}_{2}\right)=\tilde{h}_{1} @ \tilde{h}_{2}$;

(ix) $\quad\left(\tilde{h}_{1} \cup \tilde{h}_{2}\right) @\left(\tilde{h}_{1} \cap \tilde{h}_{2}\right)=\tilde{h}_{1} @ \tilde{h}_{2}$;

(x) $\quad\left(\tilde{h}_{1} \cup \widetilde{h}_{2}\right) \$\left(\tilde{h}_{1} \cap \tilde{h}_{2}\right)=\tilde{h}_{1} \$ \tilde{h}_{2}$;

(xi) $\quad\left(\tilde{h}_{1} \cup \tilde{h}_{2}\right) \#\left(\tilde{h}_{1} \cap \tilde{h}_{2}\right)=\tilde{h}_{1} \# \widetilde{h}_{2}$;

(xii) $\quad\left(\tilde{h}_{1} \cup \tilde{h}_{2}\right) *\left(\tilde{h}_{1} \cap \tilde{h}_{2}\right)=\tilde{h}_{1} * \tilde{h}_{2}$;

(xiii) $\quad\left(\tilde{h}_{1} * \tilde{h}_{2}\right) @\left(\tilde{h}_{1} * \tilde{h}_{2}\right)=\tilde{h}_{1} * \tilde{h}_{2}$;

(xiv) $\quad\left(\tilde{h}_{1} * \tilde{h}_{2}\right) \$\left(\tilde{h}_{1} * \tilde{h}_{2}\right)=\tilde{h}_{1} * \tilde{h}_{2}$.

Proof, The proofs of these results are the same as in the above proof

\section{Theorem 3.9.}

For every two $\tilde{h}_{1}$ and $\tilde{h}_{2} \in \operatorname{IVIHFE}(\mathrm{X})$, we have: 
(i) $\quad\left(\left(\tilde{h}_{1} \cup \tilde{h}_{2}\right) \oplus\left(\tilde{h}_{1} \cap \tilde{h}_{2}\right)\right) @\left(\left(\tilde{h}_{1} \cup \tilde{h}_{2}\right) \otimes\left(\tilde{h}_{1} \cap \tilde{h}_{2}\right)\right)=\tilde{h}_{1} @ \tilde{h}_{2}$;

(ii) $\quad\left(\left(\tilde{h}_{1} \cup \tilde{h}_{2}\right) \#\left(\tilde{h}_{1} \cap \tilde{h}_{2}\right)\right) \$\left(\left(\tilde{h}_{1} \cup \tilde{h}_{2}\right) @\left(\tilde{h}_{1} \cap \tilde{h}_{2}\right)\right)=\tilde{h}_{1} \$ \tilde{h}_{2}$

(iii) $\quad\left(\left(\tilde{h}_{1} \oplus \tilde{h}_{2}\right) \cup\left(\tilde{h}_{1} \otimes \tilde{h}_{2}\right)\right) @\left(\left(\tilde{h}_{1} \oplus \tilde{h}_{2}\right) \cap\left(\tilde{h}_{1} \otimes \tilde{h}_{2}\right)\right)=\tilde{h}_{1} @ \tilde{h}_{2}$;

(iv) $\quad\left(\left(\tilde{h}_{1} \oplus \tilde{h}_{2}\right) \cup\left(\tilde{h}_{1} @ \tilde{h}_{2}\right)\right) @\left(\left(\tilde{h}_{1} \otimes \tilde{h}_{2}\right) \cap\left(\tilde{h}_{1} @ \tilde{h}_{2}\right)\right)=\tilde{h}_{1} @ \tilde{h}_{2}$;

(v) $\quad\left(\left(\tilde{h}_{1} \oplus \tilde{h}_{2}\right) \cup\left(\tilde{h}_{1} \# \tilde{h}_{2}\right)\right) @\left(\left(\tilde{h}_{1} \otimes \tilde{h}_{2}\right) \cap\left(\tilde{h}_{1} \# \tilde{h}_{2}\right)\right)=\tilde{h}_{1} @ \tilde{h}_{2}$;

(vi) $\quad\left(\left(\tilde{h}_{1} \oplus \tilde{h}_{2}\right) \cup\left(\tilde{h}_{1} \$ \tilde{h}_{2}\right)\right) @\left(\left(\tilde{h}_{1} \otimes \tilde{h}_{2}\right) \cap\left(\tilde{h}_{1} \$ \tilde{h}_{2}\right)\right)=\tilde{h}_{1} @ \tilde{h}_{2}$;

(vii) $\quad\left(\left(\tilde{h}_{1} \oplus \tilde{h}_{2}\right) \cup\left(\tilde{h}_{1} @ \tilde{h}_{2}\right)\right) @\left(\left(\tilde{h}_{1} \oplus \tilde{h}_{2}\right) \cap\left(\tilde{h}_{1} \# \tilde{h}_{2}\right)\right)=\tilde{h}_{1} \$ \tilde{h}_{2}$.

Proof .In the following, we prove (i) and (iii), other results can be proved analogously.

(i) From definitions 2.3 and 3.1, we have

$\left(\left(\tilde{h}_{1} \cup \tilde{h}_{2}\right) \oplus\left(\tilde{h}_{1} \cap \tilde{h}_{2}\right)\right) @\left(\left(\tilde{h}_{1} \cup \tilde{h}_{2}\right) \otimes\left(\tilde{h}_{1} \cap \tilde{h}_{2}\right)\right)=$

$\left(\left(\tilde{h}_{1} \cup \tilde{h}_{2}\right) \oplus\left(\tilde{h}_{1} \cap \tilde{h}_{2}\right)\right)=$

$\left\{\left[\max \left(\mu_{\alpha_{1}}^{-}, \mu_{\alpha_{2}}^{-}\right), \max \left(\mu_{\alpha_{1}}^{+}, \mu_{\alpha_{2}}^{+}\right)\right],\left[\min \left(v_{\alpha_{1}}^{-}, v_{\alpha_{2}}^{-}\right), \min \left(v_{\alpha_{1}}^{+}, v_{\alpha_{2}}^{+}\right)\right] \mid \alpha_{1} \in \tilde{h}_{1}, \alpha_{2} \in \tilde{h}_{2}\right\} \oplus\left\{\left[\min \left(\mu_{\alpha_{1}}^{-}, \mu_{\alpha_{2}}^{-}\right), \min \left(\mu_{\alpha_{1}}^{+}\right.\right.\right.$, $\left.\left.\mu_{\alpha_{2}}^{+}\right)\right],\left[\max \left[\left(v_{\alpha_{1}}^{-}, v_{\alpha_{2}}^{-}\right), \max \left(v_{\alpha_{1}}^{+}, v_{\alpha_{2}}^{+}\right)\right] \mid \alpha_{1} \in \tilde{h}_{1}, \alpha_{2} \in \tilde{h}_{2}\right\}$

$=\left\{\left[\max \left(\mu_{\alpha_{1}}^{-}, \mu_{\alpha_{2}}^{-}\right)+\min \left(\mu_{\alpha_{1}}^{-}, \mu_{\alpha_{2}}^{-}\right)-\max \left(\mu_{\alpha_{1}}^{-}, \mu_{\alpha_{2}}^{-}\right) \min \left(\mu_{\alpha_{1}}^{-}, \mu_{\alpha_{2}}^{-}\right), \max \left(\mu_{\alpha_{1}}^{+}, \mu_{\alpha_{2}}^{+}\right)+\min \left(\mu_{\alpha_{1}}^{+}, \mu_{\alpha_{2}}^{+}\right)-\max \left(\mu_{\alpha_{1}}^{+}\right.\right.\right.$, $\left.\left.\mu_{\alpha_{2}}^{+}\right)+\min \left(\mu_{\alpha_{1}}^{+}, \mu_{\alpha_{2}}^{+}\right)\right],\left[\min \left(v_{\alpha_{1}}^{-}, v_{\alpha_{2}}^{-}\right) \max \left(\left(_{\alpha_{1}}^{-}, v_{\alpha_{2}}^{-}\right), \min \left(v_{\alpha_{1}}^{+}, v_{\alpha_{2}}^{+}\right) \max \left(v_{\alpha_{1}}^{+}, v_{\alpha_{2}}^{+}\right)\right]\right.$

$\left(\tilde{h}_{1} \cup \tilde{h}_{2}\right) \otimes\left(\tilde{h}_{1} \cap \tilde{h}_{2}\right)=$

$\left\{\left[\max \left(\mu_{\alpha_{1}}^{-}, \mu_{\alpha_{2}}^{-}\right), \max \left(\mu_{\alpha_{1}}^{+}, \mu_{\alpha_{2}}^{+}\right)\right],\left[\min \left[\left(v_{\alpha_{1}}^{-}, v_{\alpha_{2}}^{-}\right), \min \left(v_{\alpha_{1}}^{+}, v_{\alpha_{2}}^{+}\right)\right] \mid \alpha_{1} \in \tilde{h}_{1}, \alpha_{2} \in \tilde{h}_{2}\right\} \otimes\left\{\left[\min \left(\mu_{\alpha_{1}}^{-}, \mu_{\alpha_{2}}^{-}\right)\right.\right.\right.$, $\left.\left.\min \left(\mu_{\alpha_{1}}^{+}, \mu_{\alpha_{2}}^{+}\right)\right],\left[\max \left(\mathrm{v}_{\alpha_{1}}^{-}, v_{\alpha_{2}}^{-}\right), \max \left(v_{\alpha_{1}}^{+}, v_{\alpha_{2}}^{+}\right)\right] \mid \alpha_{1} \in \tilde{h}_{1}, \alpha_{2} \in \tilde{h}_{2}\right\}$

$=\left\{\left[\max \left(\mu_{\alpha_{1}}^{-}, \mu_{\alpha_{2}}^{-}\right) \min \left(\mu_{\alpha_{1}}^{-}, \mu_{\alpha_{2}}^{-}\right), \max \left(\mu_{\alpha_{1}}^{+}, \mu_{\alpha_{2}}^{+}\right) \min \left(\mu_{\alpha_{1}}^{+}, \mu_{\alpha_{2}}^{+}\right)\right],\left[\min \left(v_{\alpha_{1}}^{-}, v_{\alpha_{2}}^{-}\right)+\right.\right.$

$\left.\max \left(v_{\alpha_{1}}^{-}, v_{\alpha_{2}}^{-}\right)-\min \left(v_{\alpha_{1}}^{-}, v_{\alpha_{2}}^{-}\right) \max \left(v_{\alpha_{1}}^{-}, v_{\alpha_{2}}^{-}\right), \min \left(v_{\alpha_{1}}^{+}, v_{\alpha_{2}}^{+}\right)+\max \left(v_{\alpha_{1}}^{+}, v_{\alpha_{2}}^{+}\right)-\min \left(v_{\alpha_{1}}^{+}, v_{\alpha_{2}}^{+}\right) \max \left(v_{\alpha_{1}}^{+}, v_{\alpha_{2}}^{+}\right)\right] \mid \alpha_{1} \in$ $\left.\tilde{h}_{1}, \alpha_{2} \in \tilde{h}_{2}\right\}$

$\left(\left(\tilde{h}_{1} \cup \tilde{h}_{2}\right) \oplus\left(\tilde{h}_{1} \cap \tilde{h}_{2}\right)\right) @\left(\left(\tilde{h}_{1} \cup \tilde{h}_{2}\right) \otimes\left(\tilde{h}_{1} \cap \tilde{h}_{2}\right)\right)=$

$\left\{\left[\frac{\left\{\max \left(\mu_{\alpha_{1}}^{-}, \mu_{\alpha_{2}}^{-}\right)+\min \left(\mu_{\alpha_{1}}^{-}, \mu_{\alpha_{2}}^{-}\right)-\max \left(\mu_{\alpha_{1}}^{-}, \mu_{\alpha_{2}}^{-}\right) \min \left(\mu_{\alpha_{1}}^{-}, \mu_{\alpha_{2}}^{-}\right)\right\}+\max \left(\mu_{\alpha_{1}}^{-}, \mu_{\alpha_{2}}^{-}\right) \min \left(\mu_{\alpha_{1}}^{-}, \mu_{\alpha_{2}}^{-}\right)}{2}\right.\right.$,
$\left.\frac{\left\{\max \left(\mu_{\alpha_{1}}^{+}, \mu_{\alpha_{2}}^{+}\right)+\min \left(\mu_{\alpha_{1}}^{+}, \mu_{\alpha_{2}}^{+}\right)-\max \left(\mu_{\alpha_{1}}^{+}, \mu_{\alpha_{2}}^{+}\right)+\min \left(\mu_{\alpha_{1}}^{+}, \mu_{\alpha_{2}}^{+}\right)\right\}+\max \left(\mu_{\alpha_{1}}^{+}, \mu_{\alpha_{2}}^{+}\right) \min \left(\mu_{\alpha_{1}}^{+}, \mu_{\alpha_{2}}^{+}\right)}{2}\right]$,

$\left[\frac{\min \left(v_{\alpha_{1}}^{-}, v_{\alpha_{2}}^{-}\right) \max \left(v_{\alpha_{1}}^{-}, v_{\alpha_{2}}^{-}\right)+\min \left(v_{\alpha_{1}}^{-}, v_{\alpha_{2}}^{-}\right)+\max \left(v_{\alpha_{1}}^{-}, v_{\alpha_{2}}^{-}\right)-\min \left(v_{\alpha_{1}}^{-}, v_{\alpha_{2}}^{-}\right) \max \left(\left[v_{\alpha_{1}}^{-}, v_{\alpha_{2}}^{-}\right)\right.}{2}\right.$,

$\left.\frac{\min \left(v_{\alpha_{1}}^{+}, v_{\alpha_{2}}^{+}\right) \max \left(v_{\alpha_{1}}^{+}, v_{\alpha_{2}}^{+}\right)+\min \left(v_{\alpha_{1}}^{+}, v_{\alpha_{2}}^{+}\right)+\max \left(v_{\alpha_{1}}^{+}, v_{\alpha_{2}}^{+}\right)-\min \left(v_{\alpha_{1}}^{+}, v_{\alpha_{2}}^{+}\right) \max \left(v_{\alpha_{1}}^{+}, v_{\alpha_{2}}^{+}\right)}{2}\right]$

$=\left[\frac{\left\{\max \left(\mu_{\alpha_{1}}^{-}, \mu_{\alpha_{2}}^{-}\right)+\min \left(\mu_{\alpha_{1}}^{-}, \mu_{\alpha_{2}}^{-}\right)\right.}{2}, \frac{\left\{\max \left(\mu_{\alpha_{1}}^{+}, \mu_{\alpha_{2}}^{+}\right)+\min \left(\mu_{\alpha_{1}}^{+}, \mu_{\alpha_{2}}^{+}\right)\right\}}{2}\right]$,

$\left[\frac{\min \left(v_{\alpha_{1}}^{-}, v_{\alpha_{2}}^{-}\right) \max \left(v_{\alpha_{1}}^{-}, v_{\alpha_{2}}^{-}\right)+\min \left(v_{\alpha_{1}}^{-}, v_{\alpha_{2}}^{-}\right)+\max \left(v_{\alpha_{1}}^{-}, v_{\alpha_{2}}^{-}\right)-\min \left(v_{\alpha_{1}}^{-}, v_{\alpha_{2}}^{-}\right) \max \left(v_{\alpha_{1}}^{-}, v_{\alpha_{2}}^{-}\right)}{2}\right.$,

$\left.\left.\frac{\min \left(v_{\alpha_{1}}^{+}, v_{\alpha_{2}}^{+}\right) \max \left(v_{\alpha_{1}}^{+}, v_{\alpha_{2}}^{+}\right)+\min \left(v_{\alpha_{1}}^{+}, v_{\alpha_{2}}^{+}\right)+\max \left(v_{\alpha_{1}}^{+}, v_{\alpha_{2}}^{+}\right)-\min \left(v_{\alpha_{1}}^{+}, v_{\alpha_{2}}^{+}\right) \max \left(v_{\alpha_{1}}^{+}, v_{\alpha_{2}}^{+}\right)}{2}\right] \mid \alpha_{1} \in \tilde{h}_{1}, \alpha_{2} \in \tilde{h}_{2}\right\}$

$=\left\{\left[\frac{\mu_{\alpha_{1}}^{-}+\mu_{\alpha_{2}}^{-}}{2}, \frac{\mu_{\alpha_{1}}^{+}+\mu_{\alpha_{2}}^{+}}{2}\right],\left[\frac{\min \left(\left(v_{\alpha_{1}}^{-}, v_{\alpha_{2}}^{-}\right)+\max \left(v_{\alpha_{1}}^{-}, v_{\alpha_{2}}^{-}\right)\right.}{2}, \frac{\min \left(v_{\alpha_{1}}^{+}, v_{\alpha_{2}}^{+}\right)+\max \left(v_{\alpha_{1}}^{+}, v_{\alpha_{2}}^{+}\right)}{2}\right] \mid \alpha_{1} \in \tilde{h}_{1}, \alpha_{2} \in \tilde{h}_{2}\right\}$

$=\left\{\left[\frac{\mu_{\alpha_{1}}^{-}+\mu_{\alpha_{2}}^{-}}{2}, \frac{\mu_{\alpha_{1}}^{+}+\mu_{\alpha_{2}}^{+}}{2}\right],\left[\frac{v_{\alpha_{1}}^{-}+v_{\alpha_{2}}^{-}}{2}, \frac{v_{\alpha_{1}}^{+}+v_{\alpha_{2}}^{+}}{2}\right] \mid \alpha_{1} \in \tilde{h}_{1}, \alpha_{2} \in \tilde{h}_{2}\right\}$

$=\tilde{h}_{1} @ \tilde{h}_{2}$

This proves (i).

(ii) From definitions 2.3 and 3.1, we have

$\left(\left(\tilde{h}_{1} \oplus \tilde{h}_{2}\right) \cup\left(\tilde{h}_{1} \otimes \tilde{h}_{2}\right)\right) @\left(\left(\tilde{h}_{1} \oplus \tilde{h}_{2}\right) \cap\left(\tilde{h}_{1} \otimes \tilde{h}_{2}\right)\right)=\tilde{h}_{1} @ \tilde{h}_{2} ;$

$\left(\tilde{h}_{1} \oplus \tilde{h}_{2}\right) \cap\left(\tilde{h}_{1} \otimes \tilde{h}_{2}\right)=\left\{\left[\mu_{\alpha_{1}}^{-}+\mu_{\alpha_{2}}^{-}-\mu_{\alpha_{2}}^{-} \mu_{\alpha_{1}}^{-}, \mu_{\alpha_{1}}^{+}+\mu_{\alpha_{2}}^{+}-\mu_{\alpha_{2}}^{+} \mu_{\alpha_{1}}^{+}\right],\left[v_{\alpha_{2}}^{-} v_{\alpha_{1}}^{-}, v_{\alpha_{2}}^{+} v_{\alpha_{1}}^{+}\right] \mid \alpha_{1} \in \tilde{h}_{1}, \alpha_{2} \in \tilde{h}_{2}\right\} \cup$

$\left\{\left[\mu_{\alpha_{1}}^{-} \mu_{\alpha_{2}}^{-}, \mu_{\alpha_{1}}^{+} \mu_{\alpha_{2}}^{+}\right],\left[v_{\alpha_{1}}^{-}+v_{\alpha_{2}}^{-}-v_{\alpha_{1}}^{-} v_{\alpha_{2}}^{-}, v_{\alpha_{1}}^{+}+v_{\alpha_{2}}^{+}-v_{\alpha_{1}}^{+} v_{\alpha_{2}}^{+}\right] \mid \alpha_{1} \in \tilde{h}_{1}, \alpha_{2} \in \tilde{h}_{2}\right\}$ 
$=\left\{\left[\min \left(\mu_{\alpha_{1}}^{-}+\mu_{\alpha_{2}}^{-}-\mu_{\alpha_{2}}^{-} \mu_{\alpha_{1}}^{-}, \mu_{\alpha_{1}}^{-} \mu_{\alpha_{2}}^{-}\right), \min \left(\mu_{\alpha_{1}}^{+}+\mu_{\alpha_{2}}^{+}-\mu_{\alpha_{2}}^{+} \mu_{\alpha_{1}}^{+}, \mu_{\alpha_{1}}^{+} \mu_{\alpha_{2}}^{+}\right)\right]\right.$,

$\left.\left[\max \left(v_{\alpha_{2}}^{-} v_{\alpha_{1}}^{-}, v_{\alpha_{1}}^{-}+v_{\alpha_{2}}^{-}-v_{\alpha_{1}}^{-} v_{\alpha_{2}}^{-}\right), \max \left(v_{\alpha_{2}}^{+} v_{\alpha_{1}}^{+}, v_{\alpha_{1}}^{+}+v_{\alpha_{2}}^{+}-v_{\alpha_{1}}^{+} v_{\alpha_{2}}^{+}\right)\right] \mid \alpha_{1} \in \tilde{h}_{1}, \alpha_{2} \in \tilde{h}_{2}\right\}$

$=\left\{\left[\mu_{\alpha_{1}}^{-} \mu_{\alpha_{2}}^{-}, \mu_{\alpha_{1}}^{+} \mu_{\alpha_{2}}^{+}\right],\left[v_{\alpha_{1}}^{-}+v_{\alpha_{2}}^{-}-v_{\alpha_{1}}^{-} v_{\alpha_{2}}^{-}, v_{\alpha_{1}}^{+}+v_{\alpha_{2}}^{+}-v_{\alpha_{1}}^{+} v_{\alpha_{2}}^{+}\right] \mid \alpha_{1} \in \tilde{h}_{1}, \alpha_{2} \in \tilde{h}_{2}\right\}$

$\left(\tilde{h}_{1} \oplus \tilde{h}_{2}\right) \cup\left(\tilde{h}_{1} \otimes \tilde{h}_{2}\right)=\left\{\left[\mu_{\alpha_{1}}^{-}+\mu_{\alpha_{2}}^{-}-\mu_{\alpha_{2}}^{-} \mu_{\alpha_{1}}^{-}, \mu_{\alpha_{1}}^{+}+\mu_{\alpha_{2}}^{+}-\mu_{\alpha_{2}}^{+} \mu_{\alpha_{1}}^{+}\right],\left[v_{\alpha_{2}}^{-} v_{\alpha_{1}}^{-}, v_{\alpha_{2}}^{+} v_{\alpha_{1}}^{+}\right] \mid \alpha_{1} \in \tilde{h}_{1}, \alpha_{2} \in \tilde{h}_{2}\right\} \cup$

$\left\{\left[\mu_{\alpha_{1}}^{-} \mu_{\alpha_{2}}^{-}, \mu_{\alpha_{1}}^{+} \mu_{\alpha_{2}}^{+}\right],\left[v_{\alpha_{1}}^{-}+v_{\alpha_{2}}^{-}-v_{\alpha_{1}}^{-} v_{\alpha_{2}}^{-}, v_{\alpha_{1}}^{+}+v_{\alpha_{2}}^{+}-v_{\alpha_{1}}^{+} v_{\alpha_{2}}^{+}\right] \mid \alpha_{1} \in \tilde{h}_{1}, \alpha_{2} \in \tilde{h}_{2}\right\}$

$=\left\{\left[\max \left(\mu_{\alpha_{1}}^{-}+\mu_{\alpha_{2}}^{-}-\mu_{\alpha_{2}}^{-} \mu_{\alpha_{1}}^{-}, \mu_{\alpha_{1}}^{-} \mu_{\alpha_{2}}^{-}\right), \max \left(\mu_{\alpha_{1}}^{+}+\mu_{\alpha_{2}}^{+}-\mu_{\alpha_{2}}^{+} \mu_{\alpha_{1}}^{+}, \mu_{\alpha_{1}}^{+} \mu_{\alpha_{2}}^{+}\right)\right]\right.$,

$\left.\left[\min \left(v_{\alpha_{2}}^{-} v_{\alpha_{1}}^{-}, v_{\alpha_{1}}^{-}+v_{\alpha_{2}}^{-}-v_{\alpha_{1}}^{-} v_{\alpha_{2}}^{-}\right), \min \left(v_{\alpha_{2}}^{+} v_{\alpha_{1}}^{+}, v_{\alpha_{1}}^{+}+v_{\alpha_{2}}^{+}-v_{\alpha_{1}}^{+} v_{\alpha_{2}}^{+}\right)\right] \mid \alpha_{1} \in \tilde{h}_{1}, \alpha_{2} \in \tilde{h}_{2}\right\}$

$=\left\{\left[\mu_{\alpha_{1}}^{-}+\mu_{\alpha_{2}}^{-}-\mu_{\alpha_{2}}^{-} \mu_{\alpha_{1}}^{-}, \mu_{\alpha_{1}}^{+}+\mu_{\alpha_{2}}^{+}-\mu_{\alpha_{2}}^{+} \mu_{\alpha_{1}}^{+}\right],\left[v_{\alpha_{2}}^{-} v_{\alpha_{1}}^{-}, v_{\alpha_{2}}^{+} v_{\alpha_{1}}^{+}\right] \mid \alpha_{1} \in \tilde{h}_{1}, \alpha_{2} \in \tilde{h}_{2}\right\}$

$\left(\left(\left(\tilde{h}_{1} \oplus \tilde{h}_{2}\right) \cup\left(\tilde{h}_{1} \otimes \tilde{h}_{2}\right)\right) @\left(\left(\tilde{h}_{1} \oplus \tilde{h}_{2}\right) \cap\left(\tilde{h}_{1} \otimes \tilde{h}_{2}\right)\right)=\left\{\left[\frac{\mu_{\alpha_{1}}^{-} \mu_{\alpha_{2}}^{-}+\mu_{\alpha_{1}}^{-}+\mu_{\alpha_{2}}^{-}-\mu_{\alpha_{2}}^{-} \mu_{\alpha_{1}}^{-}}{2}, \frac{\mu_{\alpha_{1}}^{+} \mu_{\alpha_{2}}^{+}+\mu_{\alpha_{1}}^{+}+\mu_{\alpha_{2}}^{+}-\mu_{\alpha_{2}}^{+} \mu_{\alpha_{1}}^{+}}{2}\right]\right.\right.$,

$\left.\left[\frac{v_{\alpha_{1}}^{-}+v_{\alpha_{2}}^{-}-v_{\alpha_{1}}^{-} v_{\alpha_{2}}^{-}+v_{\alpha_{2}}^{-} v_{\alpha_{1}}^{-}}{2}, \frac{v_{\alpha_{1}}^{+}+v_{\alpha_{2}}^{+}-v_{\alpha_{1}}^{+} v_{\alpha_{2}}^{+}+v_{\alpha_{2}}^{+} v_{\alpha_{1}}^{+}}{2}\right] \mid \alpha_{1} \in \tilde{h}_{1}, \alpha_{2} \in \tilde{h}_{2}\right\}$

$=\left\{\left[\frac{\mu_{\alpha_{1}}^{-}+\mu_{\alpha_{2}}^{-}}{2}, \frac{\mu_{\alpha_{1}+\mu_{\alpha_{2}}^{+}}^{+}}{2}\right],\left[\frac{v_{\alpha_{1}}^{-}+v_{\alpha_{2}}^{-}}{2}, \frac{v_{\alpha_{1}}^{+}+v_{\alpha_{2}}^{+}}{2}\right] \mid \alpha_{1} \in \tilde{h}_{1}, \alpha_{2} \in \tilde{h}_{2}\right\}$

Hence,$\left(\left(\left(\tilde{h}_{1} \oplus \tilde{h}_{2}\right) \cup\left(\tilde{h}_{1} \otimes \tilde{h}_{2}\right)\right) @\left(\left(\tilde{h}_{1} \oplus \tilde{h}_{2}\right) \cap\left(\tilde{h}_{1} \otimes \tilde{h}_{2}\right)\right)=\tilde{h}_{1} @ \tilde{h}_{2}\right.$ This proves (ii). 


\section{Conclusion}

In this paper, we have defined four new operations on interval valued intuitionistic hesitant fuzzy sets which involve different defining functions. Some related results have been proved and the characteristics of the interval valued intuitionistic hesitant fuzzy sets have been brought out..

\section{Acknowledgements}

The authors are very grateful to the anonymous referees for their insightful and constructive comments and suggestions, which have been very helpful in improving the paper.

\section{REFERENCES}

[1] Zhiming Zhang ," Interval-Valued Intuitionistic Hesitant Fuzzy Aggregation Operators and Their Application in Group Decision-Making", Journal of Applied Mathematics Volume 2013 pages ,http://dx.doi.org/10.1155/2013/670285.

[2] Zadeh .L A , Fuzzy sets. Information and Control 8(3) ,1965,pp.338-353.

[3] Zadeh L A , The concept of a linguistic variable and its application to approximate reasoning-I.Information Sciences 8(3),1975, pp.199-249.

[4] Atanassov K T , Intuitionistic fuzzy sets. Fuzzy Sets and Systems 20(1),1986, pp.87-96.

[5] Atanassov K T , Intuitionistic fuzzy sets. Springer Physica-Verlag, Heidelberg, 1999.

[6] K. T. Atanassov and G. Gargov, Interval valued intuitionistic fuzzy sets, Fuzzy Sets and Systems, Vol. 31, Issue 3, 1989, pp. $343-349$
[7] Dubois D, Prade H , Fuzzy sets and systems: theory and applications. Academic Press, NewYork,1980.

[8] Karnik N N, Mendel J M , Operations on type-2 fuzzy sets. Fuzzy Sets and Systems 122(2),2001,pp.327-348.

[9] Torra V, Narukawa Y, On hesitant fuzzy sets and decision. The 18th IEEE international Conference on Fuzzy Systems, Jeju Island, Korea,2009, pp.1378-1382.

[10] Torra $\mathrm{V}$,Hesitant fuzzy sets. International Journal of Intelligent Systems 25(6),2010, pp.529-539.

[11] Xia M, Xu Z S Hesitant fuzzy information aggregation in decision making. International Journal of Approximate Reasoning 52(3),2011, pp.395-407.

[12] Xia M M, Xu Z S, Chen N, Induced aggregation under confidence levels. International Journal of Uncertainty, Fuzziness and Knowledge-Based Systems 19(2),2011, pp.201-227

[13] Xu Z S, Xia M M ,Distance and similarity measures on hesitant fuzzy sets. Information Sciences 181(11),2011, pp.2128-2138.

[14] Xu Z S, Xia M M, On distance and correlation measures of hesitant fuzzy information. Inter-national Journal of Intelligent Systems 26(5),2011,pp. 410-425.

3 (2013), Article ID 670285, 33

[15] Xu ZS ,Methods for aggregating interval-valued intuitionistic fuzzy information and their application to decision making. Control Decis,22(2) ,2007, pp.215-219.

[16] Wei GW, Wang XR ,Some geometric aggregation operators based on interval-valued intuitionistic fuzzy sets and their application to group decision making, In Proceedings of the international conference on computational intelligence and security. Washington, DC, USA: IEEE Computer Society Press, 2007, pp. 495-499.

[17] $\mathrm{Xu} \mathrm{ZS,} \mathrm{Chen} \mathrm{J}$, On geometric aggregation over interval-valued intuitionistic fuzzy information., In Proceedings of the fourth international conference on fuzzy systems and knowledge discovery. Washington, DC, USA: IEEE Computer Society Press,,2007,pp. 466-471. 